\title{
Higher Spin Alternating Sign Matrices
}

\author{
Roger E. Behrend and Vincent A. Knight \\ School of Mathematics, Cardiff University, \\ Cardiff, CF24 4AG, UK \\ behrendr@cardiff.ac.uk, knightva@cardiff.ac.uk
}

Submitted: Aug 28, 2007; Accepted: Nov 25, 2007; Published: Nov 30, 2007

Mathematics Subject Classifications: 05A15, 05B20, 52B05, 52B11, 82B20, 82B23

\begin{abstract}
We define a higher spin alternating sign matrix to be an integer-entry square matrix in which, for a nonnegative integer $r$, all complete row and column sums are $r$, and all partial row and column sums extending from each end of the row or column are nonnegative. Such matrices correspond to configurations of spin $r / 2$ statistical mechanical vertex models with domain-wall boundary conditions. The case $r=1$ gives standard alternating sign matrices, while the case in which all matrix entries are nonnegative gives semimagic squares. We show that the higher spin alternating sign matrices of size $n$ are the integer points of the $r$-th dilate of an integral convex polytope of dimension $(n-1)^{2}$ whose vertices are the standard alternating sign matrices of size $n$. It then follows that, for fixed $n$, these matrices are enumerated by an Ehrhart polynomial in $r$.

Keywords: alternating sign matrix, semimagic square, convex polytope, higher spin vertex model
\end{abstract}




\section{Introduction}

Alternating sign matrices are mathematical objects with intriguing combinatorial properties and important connections to mathematical physics, and the primary aim of this paper is to introduce natural generalizations of these matrices which also seem to display interesting such properties and connections.

Alternating sign matrices were first defined in [50], and the significance of their connection with mathematical physics first became apparent in [47], in which a determinant formula for the partition function of an integrable statistical mechanical model, and a simple correspondence between configurations of that model and alternating sign matrices, were used to prove the validity of a previously-conjectured enumeration formula. For reviews of this and related areas, see for example $[16,17,57,72]$. Such connections with statistical mechanical models have since been used extensively to derive formulae for further cases of refined, weighted or symmetry-class enumeration of alternating sign matrices, as done for example in $[22,48,56,71]$.

The statistical mechanical model used in all of these cases is the integrable six-vertex model (with certain boundary conditions), which is intrinsically related to the spin $1 / 2$, or two dimensional, irreducible representation of the Lie algebra $s l(2, \mathbb{C})$. For a review of this area, see for example [39]. In this paper, we consider configurations of statistical mechanical vertex models (again with certain boundary conditions) related to the spin $r / 2$ representation of $\operatorname{sl}(2, \mathbb{C})$, for all nonnegative integers $r$, these being in simple correspondence with matrices which we term higher spin alternating sign matrices. Determinant formulae for the partition functions of these models have already been obtained in [18], thus for example answering Question 22 of [48] on whether such formulae exist.

Although we were originally motivated to consider higher spin alternating sign matrices through this connection with statistical mechanical models, these matrices are natural generalizations of standard alternating sign matrices in their own right, and appear to have important combinatorial properties. Furthermore, they generalize not only standard alternating sign matrices, but also other much-studied combinatorial objects, namely semimagic squares.

Semimagic squares are simply nonnegative integer-entry square matrices in which all complete row and column sums are equal. They are thus the integer points of the integer dilates of the convex polytope of nonnegative real-entry, fixed-size square matrices in which all complete row and column sums are 1, a fact which leads to enumeration results for the case of fixed size. For reviews of this area, see for example [7, Ch. 6] or [63, Sec. 4.6]. In this paper, we introduce an analogous convex polytope, which was independently defined 
and studied in [65], and for which the integer points of the integer dilates are the higher spin alternating sign matrices of fixed size.

We define higher spin alternating sign matrices in Section 2, after which this paper then divides into two essentially independent parts: Sections 3, 4 and 5, and Sections 6, 7 and 8. In Sections 3, 4 and 5, we define and discuss various combinatorial objects which are in bijection with higher spin alternating sign matrices, and which generalize previouslystudied objects in bijection with standard alternating sign matrices. In Sections 6, 7 and 8, we define and study the convex polytope which is related to higher spin alternating sign matrices, and we obtain certain enumeration formulae for the case of fixed size. We then end the paper in Section 9 with a discussion of possible further research.

Finally in this introduction, we note that standard alternating sign matrices are related to many further fascinating results and conjectures in combinatorics and mathematical physics beyond those already mentioned or directly relevant to this paper. For example, in combinatorics it is known that the numbers of standard alternating sign matrices, descending plane partitions, and totally symmetric self-complementary plane partitions of certain sizes are all equal, but no bijective proofs of these equalities have yet been found. Moreover, further equalities between the cardinalities of certain subsets of these three objects have been conjectured, some over two decades ago, and many of these remain unproved. See for example [3, 4, 26, 27, 41, 42, 51, 52]. Meanwhile, in mathematical physics, extensive work has been done recently on so-called Razumov-Stroganov-type results and conjectures. These give surprising equalities between numbers of certain alternating sign matrices or plane partitions, and entries of eigenvectors related to certain statistical mechanical models. See for example [24, 25] and references therein.

Notation. Throughout this paper, $\mathbb{P}$ denotes the set of positive integers, $\mathbb{N}$ denotes the set of nonnegative integers, $[m, n]$ denotes the set $\{m, m+1, \ldots, n\}$ for any $m, n \in \mathbb{Z}$, with $[m, n]=\emptyset$ for $n<m$, and $[n]$ denotes the set $[1, n]$ for any $n \in \mathbb{Z}$. The notation $(0,1)_{\mathbb{R}}$ and $[0,1]_{\mathbb{R}}$ will be used for the open and closed intervals of real numbers between 0 and 1 . For a finite set $T,|T|$ denotes the cardinality of $T$.

\section{Higher Spin Alternating Sign Matrices}

In this section, we define higher spin alternating sign matrices, describe some of their basic properties, introduce an example, and give an enumeration table.

For $n \in \mathbb{P}$ and $r \in \mathbb{N}$, let the set of higher spin alternating sign matrices of size $n$ with line sum $r$ be 


$$
\begin{aligned}
& \operatorname{ASM}(n, r):=
\end{aligned}
$$

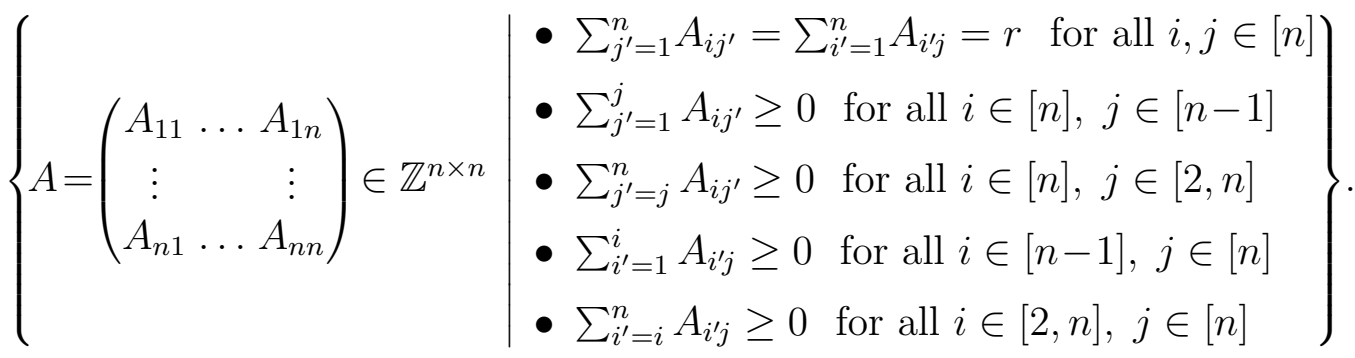

In other words, $\operatorname{ASM}(n, r)$ is the set of $n \times n$ integer-entry matrices for which all complete row and column sums are $r$, and all partial row and column sums extending from each end of the row or column are nonnegative. As will be explained in Section 3, a line sum of $r$ corresponds to a spin of $r / 2$. The set $\operatorname{ASM}(n, r)$ can also be written as

$$
\begin{aligned}
& \operatorname{ASM}(n, r)=
\end{aligned}
$$

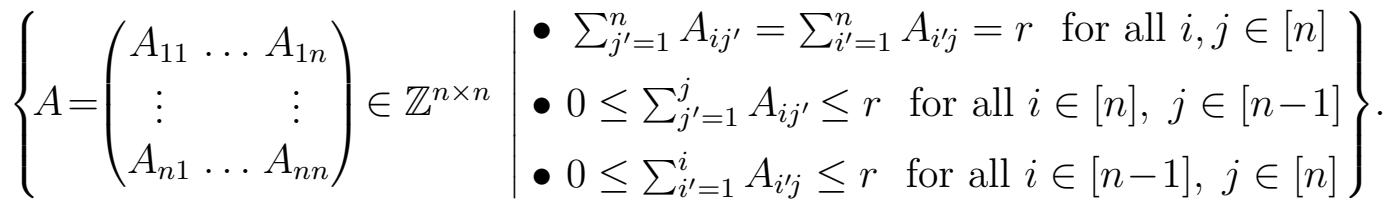

It follows that each entry of any matrix of $\operatorname{ASM}(n, r)$ is between $-r$ and $r$, and that if the entry is in the first or last row or column, then it is between 0 and $r$.

A running example will be the matrix

$$
A=\left(\begin{array}{ccccc}
0 & 1 & 1 & 0 & 0 \\
1 & -1 & 0 & 2 & 0 \\
0 & 1 & 1 & -2 & 2 \\
1 & 0 & 0 & 1 & 0 \\
0 & 1 & 0 & 1 & 0
\end{array}\right) \in \operatorname{ASM}(5,2)
$$

Defining

$$
\operatorname{SMS}(n, r):=\left\{A \in \operatorname{ASM}(n, r) \mid A_{i j} \geq 0 \text { for each } i, j \in[n]\right\},
$$

it can be seen that this is the set of semimagic squares of size $n$ with line sum $r$, i.e., nonnegative integer-entry $n \times n$ matrices in which all complete row and column sums are $r$. For example, $\operatorname{SMS}(n, 1)$ is the set of $n \times n$ permutation matrices, so that

$$
|\operatorname{SMS}(n, 1)|=n !
$$

Early studies of semimagic squares appear in [2, 49]. For further information and references, see for example [7, Ch. 6], [32], [61], [62], [63, Sec. 4.6] and [64, Sec. 5.5]. 
It can also be seen that $\operatorname{ASM}(n, 1)$ is the set of standard alternating sign matrices of size $n$, i.e., $n \times n$ matrices in which each entry is 0,1 or -1 , each row and column contains at least one nonzero entry, and along each row and column the nonzero entries alternate in sign, starting and finishing with a 1 . Standard alternating sign matrices were first defined and studied in [50,51]. For further information, connections to related subjects, and references see for example $[16,17,25,55,57,72]$.

We refer to $\operatorname{ASM}(n, r)$ as a set of 'higher spin alternating sign matrices' for any $n \in \mathbb{P}$ and $r \in \mathbb{N}$, although we realize that this could be slightly misleading since the 'alternating sign' property applies only to the standard case $r=1$, and the spin $r / 2$ is only 'higher' for cases with $r \geq 2$. Nevertheless, we still feel that this is the most natural choice of terminology.

Some cardinalities of $\operatorname{ASM}(n, r)$, many of them computer-generated, are shown in Table 1.

\begin{tabular}{r|ccccc} 
& $r=0$ & 1 & 2 & 3 & 4 \\
\hline$n=1$ & 1 & 1 & 1 & 1 & 1 \\
2 & 1 & 2 & 3 & 4 & 5 \\
3 & 1 & 7 & 26 & 70 & 155 \\
4 & 1 & 42 & 628 & 5102 & 28005 \\
5 & 1 & 429 & 41784 & 1507128 & 28226084 \\
6 & 1 & 7436 & 7517457 & 1749710096 & 152363972022
\end{tabular}

Table 1: $|\operatorname{ASM}(n, r)|$ for $n \in[6], r \in[0,4]$.

Apart from the trivial formulae $|\operatorname{ASM}(n, 0)|=1$ (since $\operatorname{ASM}(n, 0)$ contains only the $n \times n$ zero matrix), $|\operatorname{ASM}(1, r)|=1$ (since $\operatorname{ASM}(1, r)=\{(r)\})$, and $|\operatorname{ASM}(2, r)|=r+1$ (since $\left.\operatorname{ASM}(2, r)=\left\{\left(\begin{array}{cc}i & r-i \\ r-i & i\end{array}\right) \mid i \in[0, r]\right\}=\operatorname{SMS}(2, r)\right)$, the only previously-known formula for a special case of $|\operatorname{ASM}(n, r)|$ is

$$
|\operatorname{ASM}(n, 1)|=\prod_{i=0}^{n-1} \frac{(3 i+1) !}{(n+i) !}
$$

for standard alternating sign matrices with any $n \in \mathbb{P}$. This formula was conjectured in $[50,51]$, and eventually proved, using different methods, in [70] and [47]. It has also been proved using a further method in [35], and, using a method related to that of [47], in $[22]$. 


\section{Edge Matrix Pairs and Higher Spin Vertex Model Configurations}

In this section, we show that there is a simple bijection between higher spin alternating sign matrices and configurations of higher spin statistical mechanical vertex models with domain-wall boundary conditions, and we discuss some properties of these vertex models.

For $n \in \mathbb{P}$ and $r \in \mathbb{N}$, define the set of edge matrix pairs as

$$
\begin{aligned}
\operatorname{EM}(n, r) & := \\
\{(H, V) & =\left(\left(\begin{array}{ccc}
H_{10} & \ldots & H_{1 n} \\
\vdots & & \vdots \\
H_{n 0} & \ldots & H_{n n}
\end{array}\right),\left(\begin{array}{ccc}
V_{01} & \ldots & V_{0 n} \\
\vdots & & \vdots \\
V_{n 1} & \ldots & V_{n n}
\end{array}\right)\right) \in[0, r]^{n \times(n+1)} \times[0, r]^{(n+1) \times n} \mid \\
H_{i 0} & \left.=V_{0 j}=0, H_{i n}=V_{n j}=r, H_{i, j-1}+V_{i j}=V_{i-1, j}+H_{i j}, \text { for all } i, j \in[n]\right\} .
\end{aligned}
$$

We shall refer to $H$ as a horizontal edge matrix and $V$ as a vertical edge matrix. It can be checked that there is a bijection between $\operatorname{ASM}(n, r)$ and $\operatorname{EM}(n, r)$ in which the edge matrix pair $(H, V)$ which corresponds to the higher spin alternating sign matrix $A$ is given by

$$
\begin{aligned}
& H_{i j}=\sum_{j^{\prime}=1}^{j} A_{i j^{\prime}}, \quad \text { for each } i \in[n], j \in[0, n] \\
& V_{i j}=\sum_{i^{\prime}=1}^{i} A_{i^{\prime} j}, \quad \text { for each } i \in[0, n], j \in[n],
\end{aligned}
$$

and inversely,

$$
A_{i j}=H_{i j}-H_{i, j-1}=V_{i j}-V_{i-1, j}, \text { for each } i, j \in[n] \text {. }
$$

Thus, $H$ is the column sum matrix and $V$ is the row sum matrix of $A$. The correspondence between standard alternating sign matrices and edge matrix pairs was first identified in $[59]$.

It can be seen that for each $(H, V) \in \operatorname{EM}(n, r)$ and $i, j \in[0, n], \sum_{i^{\prime}=1}^{n} H_{i^{\prime} j}=j r$ and $\sum_{j^{\prime}=1}^{n} V_{i j^{\prime}}=i r$, so that

$$
\sum_{i, j=1}^{n} H_{i j}=\sum_{i, j=1}^{n} V_{i j}=n(n+1) r / 2 .
$$


The edge matrix pair which corresponds to the running example (3) is

$$
(H, V)=\left(\left(\begin{array}{llllll}
0 & 0 & 1 & 2 & 2 & 2 \\
0 & 1 & 0 & 0 & 2 & 2 \\
0 & 0 & 1 & 2 & 0 & 2 \\
0 & 1 & 1 & 1 & 2 & 2 \\
0 & 0 & 1 & 1 & 2 & 2
\end{array}\right),\left(\begin{array}{lllll}
0 & 0 & 0 & 0 & 0 \\
0 & 1 & 1 & 0 & 0 \\
1 & 0 & 1 & 2 & 0 \\
1 & 1 & 2 & 0 & 2 \\
2 & 1 & 2 & 1 & 2 \\
2 & 2 & 2 & 2 & 2
\end{array}\right)\right)
$$

A configuration of a spin $r / 2$ statistical mechanical vertex model on an $n \times n$ square with domain-wall boundary conditions is the assignment, for any $(H, V) \in \operatorname{EM}(n, r)$, of the horizontal edge matrix entry $H_{i j}$ to the horizontal edge between lattice points $(i, j)$ and $(i, j+1)$, for each $i \in[n], j \in[0, n]$, and the vertical edge matrix entry $V_{i j}$ to the vertical edge between lattice points $(i, j)$ and $(i+1, j)$, for each $i \in[0, n], j \in[n]$. Throughout this paper, we use the conventions that the rows and columns of the lattice are numbered in increasing order from top to bottom, and from left to right, and that $(i, j)$ denotes the point in row $i$ and column $j$, i.e., we use matrix-type labeling of lattice points. The assignment of edge matrix entries to lattice edges is shown diagrammatically in Figure 1, and the vertex model configuration for the example of (11) is shown in Figure 2. The term domain-wall boundary conditions refers to the assignment of 0 to each edge on the left and upper boundaries of the square, and of $r$ to each edge on the lower and right boundaries of the square, i.e., to the conditions $H_{i 0}=V_{0 j}=0$ and $H_{i n}=V_{n j}=r$ of (7). The correspondence between standard alternating sign matrices and configurations of a vertex model with domain-wall boundary conditions was first identified in [33].

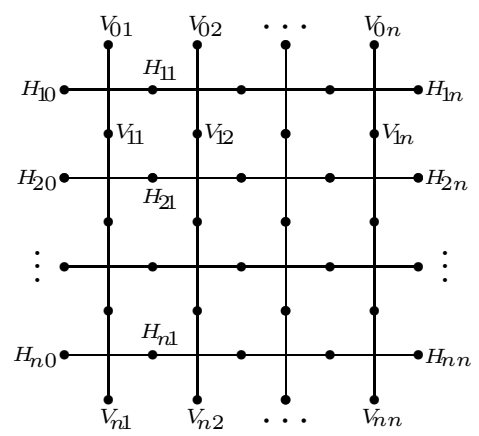

Figure 1: Assignment of edge matrix entries to lattice edges.

We note that in depicting vertex model configurations, it is often standard for certain numbers of directed arrows, rather than integers in $[0, r]$, to be assigned to lattice edges. For example, for the case $r=1$, a configuration could be depicted by assigning a leftward or rightward arrow to the horizontal edge from $(i, j)$ to $(i, j+1)$ for $H_{i j}=0$ or $H_{i j}=1$ respectively, and assigning a downward or upward arrow to the vertical edge between $(i, j)$ 


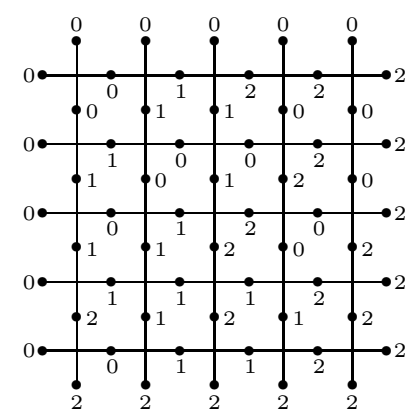

Figure 2: Vertex model configuration for the running example.

and $(i+1, j)$ for $V_{i j}=0$ or $V_{i j}=1$ respectively. The condition $H_{i, j-1}+V_{i j}=V_{i-1, j}+H_{i j}$ of $(7)$ then corresponds to arrow conservation at each lattice point (i.e., that the numbers of arrows into and out of each point are equal), while the domain-wall boundary conditions correspond to the fact that all arrows on the horizontal or vertical boundaries of the square point inwards or outwards respectively.

It is also convenient to define the set of vertex types, for a spin $r / 2$ statistical mechanical vertex model, as

$$
\mathcal{V}(r):=\left\{\left(h, v, h^{\prime}, v^{\prime}\right) \in[0, r]^{4} \mid h+v=h^{\prime}+v^{\prime}\right\}
$$

A vertex type $\left(h, v, h^{\prime}, v^{\prime}\right)$ is depicted as $h \cdot \underbrace{v^{\prime}}_{v} \cdot h^{\prime}$, and it can be seen that for the vertex model configuration associated with $(H, V) \in \operatorname{EM}(n, r)$, the lattice point $(i, j)$ is associated with the vertex type $\left(H_{i, j-1}, V_{i j}, H_{i j}, V_{i-1, j}\right) \in \mathcal{V}(r)$, for each $i, j \in[n]$.

The vertex types of $\mathcal{V}(2)$ are shown in Figure 3, where (1)-(19) will be used as labels. The vertex types of $\mathcal{V}(1)$ are (1)-(5) and (10) of Figure 3.

For any $r \in \mathbb{N}, \mathcal{V}(r)$ can be expressed as the disjoint unions

$$
\begin{aligned}
\mathcal{V}(r)= & \bigcup_{s=0}^{2 r}\left\{\left(h, s-h, h^{\prime}, s-h^{\prime}\right) \mid h, h^{\prime} \in[\max (0, s-r), \min (r, s)]\right\} \\
= & \left\{\left(h, v, h^{\prime}, h+v-h^{\prime}\right) \mid h, v, h^{\prime} \in[0, r], h \leq h^{\prime} \leq v\right\} \cup \\
& \left\{\left(h, v, h+v-v^{\prime}, v^{\prime}\right) \mid h, v, v^{\prime} \in[0, r], v<v^{\prime}<h\right\} \cup \\
& \left\{\left(h, h^{\prime}+v^{\prime}-h, h^{\prime}, v^{\prime}\right) \mid h, h^{\prime}, v^{\prime} \in[0, r], h^{\prime}<h \leq v^{\prime}\right\} \cup \\
& \left\{\left(h^{\prime}+v^{\prime}-v, v, h^{\prime}, v^{\prime}\right) \mid v, h^{\prime}, v^{\prime} \in[0, r], v^{\prime} \leq v<h^{\prime}\right\},
\end{aligned}
$$




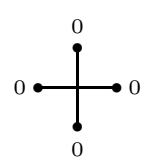

(1)

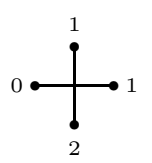

(7)

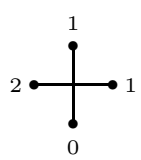

(13)

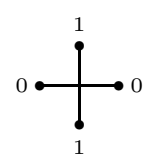

(2)

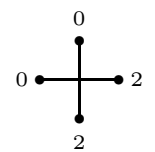

(8)

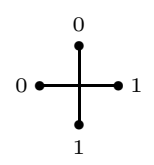

(3)

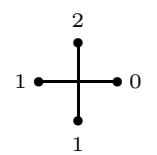

(9)

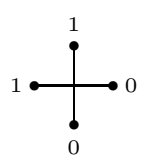

(4)

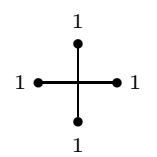

(10)

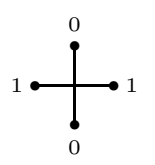

(5)

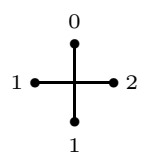

(11)

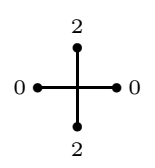

(6)

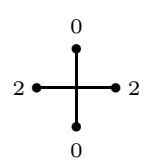

(14)

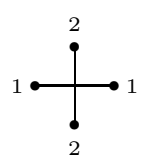

(15)

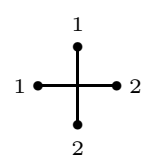

(16)

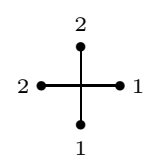

(17)

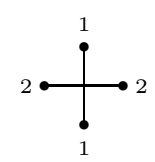

(18)

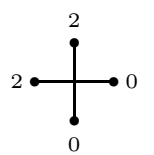

(12)

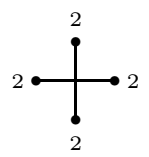

(19)

Figure 3: The 19 vertex types of $\mathcal{V}(2)$.

so that

$$
|\mathcal{V}(r)|=2 \sum_{s=1}^{r} s^{2}+(r+1)^{2}=\left(\begin{array}{c}
r+1 \\
3
\end{array}\right)+2\left(\begin{array}{c}
r+2 \\
3
\end{array}\right)+\left(\begin{array}{c}
r+3 \\
3
\end{array}\right)=(r+1)\left(2 r^{2}+4 r+3\right) / 3 .
$$

It can be seen, using (4) and (9), that a spin $r / 2$ vertex model configuration corresponds to a semimagic square with line sum $r$ if and only if each of its vertex types is in $\mathcal{V}_{\mathrm{S}}(r):=$ $\left\{\left(h, v, h^{\prime}, v^{\prime}\right) \in \mathcal{V}(r) \mid h \leq h^{\prime}\right.$ (and $\left.\left.v^{\prime} \leq v\right)\right\}$. For example, $\mathcal{V}_{\mathrm{S}}(1)$ consists of $(1)-(3),(5)$ and (10) of Figure 3, and $\mathcal{V}_{\mathrm{S}}(2)$ consists of (1)-(3), (5)-(8), (10), (11), (14)-(16), (18) and (19) of Figure 3.

By imposing the condition $h \leq h^{\prime}$ on the two disjoint unions of (13), which in the second case leaves just the first and fourth sets, it follows that $\left|\mathcal{V}_{\mathrm{S}}(r)\right|=\sum_{s=1}^{r+1} s^{2}=\left(\begin{array}{c}r+2 \\ 3\end{array}\right)+$ $\left(\begin{array}{c}r+3 \\ 3\end{array}\right)=(r+1)(r+2)(2 r+3) / 6$.

For a spin $r / 2$ statistical mechanical vertex model, a Boltzmann weight

$$
W\left(r, x, h, v, h^{\prime}, v^{\prime}\right) \in \mathbb{C}
$$

is defined for each $\left(h, v, h^{\prime}, v^{\prime}\right) \in \mathcal{V}(r)$. Here, $x$ is a complex variable, often called the spectral parameter.

For such a model on an $n$ by $n$ square with domain-wall boundary conditions, and an 
$n \times n$ matrix $z$ with entries $z_{i j} \in \mathbb{C}$ for $i, j \in[n]$, the partition function is

$$
Z(n, r, z):=\sum_{(H, V) \in \operatorname{EM}(n, r)} \prod_{i, j=1}^{n} W\left(r, z_{i j}, H_{i, j-1}, V_{i j}, H_{i j}, V_{i-1, j}\right) .
$$

Values of $Z(n, r, z)$ therefore give certain weighted enumerations of the higher spin alternating sign matrices of $\operatorname{ASM}(n, r)$. It follows that if there exists $u_{r}^{\mathrm{A}} \in \mathbb{C}$ such that

$$
W\left(r, u_{r}^{\mathrm{A}}, h, v, h^{\prime}, v^{\prime}\right)=1 \quad \text { for each }\left(h, v, h^{\prime}, v^{\prime}\right) \in \mathcal{V}(r),
$$

then

$$
\left.Z(n, r, z)\right|_{\text {each } z_{i j}=u_{r}^{\mathrm{A}}}=|\operatorname{ASM}(n, r)|,
$$

and that if there exists $u_{r}^{\mathrm{S}} \in \mathbb{C}$ such that

$$
W\left(r, u_{r}^{\mathrm{s}}, h, v, h^{\prime}, v^{\prime}\right)=\left\{\begin{array}{l}
1, h \leq h^{\prime} \\
0, h>h^{\prime}
\end{array} \quad \text { for each }\left(h, v, h^{\prime}, v^{\prime}\right) \in \mathcal{V}(r)\right.
$$

then

$$
\left.Z(n, r, z)\right|_{\text {each } z_{i j}=u_{r}^{\mathrm{S}}}=|\operatorname{SMS}(n, r)|
$$

The Boltzmann weights (15) are usually assumed to satisfy the Yang-Baxter equation and certain other properties. See for example [5, Ch. $8 \&$ 9] and [39, Ch. 1 \& 2]. Such weights can then be described as integrable, and are related to the spin $r / 2$ representation, i.e., the irreducible representation with highest weight $r$ and dimension $r+1$, of the simple Lie algebra $\operatorname{sl}(2, \mathbb{C})$, or its affine counterpart. See for example [36, 37, 39]. Each value $i \in[0, r]$, as taken by $h, v, h^{\prime}$ and $v^{\prime}$ in $(15)$, can thus be associated with an $\operatorname{sl}(2, \mathbb{C})$ weight $2 i-r$. In physics contexts, it is also natural to associate each $i \in[0, r]$ with a spin value $i-r / 2$. Integrable Boltzmann weights with $r=1$ are related to the defining spin $1 / 2$ representation of $s l(2, \mathbb{C})$, and lead to integrable six-vertex or square ice statistical mechanical models, which are associated with the $X X Z$ spin chain. Furthermore, integrable Boltzmann weights for $r>1$ can be obtained from those for $r=1$ using a procedure known as fusion. See for example [46]. Integrable Boltzmann weights for $r=2$ are also obtained more directly in $[40,60,69]$.

For integrable Boltzmann weights, and for any $x=\left(x_{1}, \ldots, x_{n}\right), y=\left(y_{1}, \ldots, y_{n}\right) \in \mathbb{C}^{n}$ with each having distinct entries, it can be shown that

$$
\left.Z(n, r, z)\right|_{\text {each } z_{i j}=x_{i}-y_{j}}=F(n, r, x, y) \operatorname{det} M(n, r, x, y)
$$

where $M(n, r, x, y)$ is an $n r \times n r$ matrix with entries $M(n, r, x, y)_{(i, k),(j, l)}=\phi\left(k-l, x_{i}-y_{j}\right)$ for each $(i, k),(j, l) \in[n] \times[r]$, and $F$ and $\phi$ are relatively simple, explicitly-known functions. This determinant formula for the partition function is proved for $r=1$ in [43, 44], using 
results of [45], and for $r>1$ in [18], using the $r=1$ result and the fusion procedure. The formula for $r=1$ is also proved in [11], using a method different from that of [43, 44], while that for $r>1$ was obtained independently of [18], but using a similar fusion method, in $[8]$.

If any entries of $x$, or any entries of $y$, are equal, then $F(n, r, x, y)$ has a singularity, and det $M(n, r, x, y)=0$. However, by taking an appropriate limit as the entries become equal, as done in [44] for $r=1$ and [18] for $r>1$, a valid alternative formula involving the determinant of an $n r \times n r$ matrix whose entries are derivatives of the function $\phi$ can be obtained. For the completely homogeneous case in which all entries of $x$ are equal, and all entries of $y$ are equal, with a difference $u$ between the entries of $x$ and $y$, this matrix has entries $\frac{d^{i+j-2}}{d u^{i+j-2}} \phi(k-l, u)$ for each $(i, k),(j, l) \in[n] \times[r]$.

For the case $r=1$, there exists $u_{1}^{\mathrm{A}}$ such that integrable Boltzmann weights satisfy (17), so that (18) can be applied together with a determinant formula. This is done in [47] and [22] in order to prove (6). In [47], a choice of $x$ and $y$ which depend on a parameter $\epsilon$ is used, in which $x$ and $y$ each have distinct entries for $\epsilon \neq 0$, and $x_{i}-y_{j}=u_{1}^{\mathrm{A}}$ for $\epsilon=0$ and each $i, j \in[n]$. The formula (21) is then applied with $\epsilon \neq 0$, the resulting determinant is evaluated as a product form, and finally the limit $\epsilon \rightarrow 0$ is taken, giving the RHS of (6). In [22], a determinant formula for the completely homogeneous case is applied at the outset, and the relation between Hankel determinants and orthogonal polynomials, together with known properties of the Continuous Hahn orthogonal polynomials, are then used to evaluate the resulting determinant, giving the RHS of (6).

For cases with $r>1$, if there exist values $u_{r}^{\mathrm{A}}$ or $u_{r}^{\mathrm{S}}$ such that (17) or (19) are satisfied for integrable Boltzmann weights, then methods similar to those used for $r=1$ could be applied in an attempt to obtain formulae for $|\operatorname{ASM}(n, r)|$ or $|\operatorname{SMS}(n, r)|$ for fixed $r$ and variable $n$. However, our preliminary investigations suggest that such $u_{r}^{\mathrm{A}}$ and $u_{r}^{\mathrm{S}}$ do not exist for integrable Boltzmann weights with $r>1$.

\section{Lattice Paths}

In this section, we show that there is also a bijection between higher spin alternating sign matrices and certain sets of lattice paths.

For $n \in \mathbb{P}$ and $r \in \mathbb{N}$, let $\operatorname{LP}(n, r)$ be the set of all sets $P$ of $n r$ directed lattice paths such that

- For each $i \in[n], P$ contains $r$ paths which begin by passing from $(n+1, i)$ to $(n, i)$ and end by passing from $(i, n)$ to $(i, n+1)$. 
- Each step of each path of $P$ is either $(-1,0)$ or $(0,1)$.

- Different paths of $P$ do not cross.

- No more than $r$ paths of $P$ pass along any edge of the lattice.

It can be checked that there is a bijection between $\operatorname{EM}(n, r)$ (and hence $\operatorname{ASM}(n, r)$ ) and $\mathrm{LP}(n, r)$ in which the edge matrix pair $(H, V)$ which corresponds to the path set $P$ is given simply by

$$
\begin{aligned}
H_{i j}= & \text { number of paths of } P \text { which pass from }(i, j) \\
& \text { to }(i, j+1), \text { for each } i \in[n], j \in[0, n] \\
V_{i j}= & \text { number of paths of } P \text { which pass from }(i+1, j) \\
& \text { to }(i, j), \text { for each } i \in[0, n], j \in[n] .
\end{aligned}
$$

For the inverse mapping from $(H, V)$ to $P,(22)$ is used to assign appropriate numbers of path segments to the horizontal and vertical edges of the lattice, and at each $(i, j) \in$ $[n] \times[n]$, the $H_{i, j-1}+V_{i j}=V_{i-1, j}+H_{i j}$ segments on the four neighboring edges are linked without crossing through $(i, j)$ according to the rules that

- If $H_{i j}=V_{i j}$ (and $H_{i, j-1}=V_{i-1, j}$ ), then $H_{i, j-1}$ paths pass from $(i, j-1)$ to $(i-1, j)$, and $H_{i j}$ paths pass from $(i+1, j)$ to $(i, j+1)$.

- If $H_{i j}>V_{i j}$ (and $H_{i, j-1}>V_{i-1, j}$ ), then $V_{i-1, j}$ paths pass from $(i, j-1)$ to $(i-1, j), H_{i j}-V_{i j}=H_{i, j-1}-V_{i-1, j}$ paths pass from $(i, j-1)$ to $(i, j+1)$, and $V_{i j}$ paths pass from $(i+1, j)$ to $(i, j+1)$.

- If $V_{i j}>H_{i j}$ (and $\left.V_{i-1, j}>H_{i, j-1}\right)$, then $H_{i, j-1}$ paths pass from $(i, j-1)$ to $(i-1, j), V_{i j}-H_{i j}=V_{i-1, j}-H_{i, j-1}$ paths pass from $(i+1, j)$ to $(i-1, j)$, and $H_{i j}$ paths pass from $(i+1, j)$ to $(i, j+1)$.

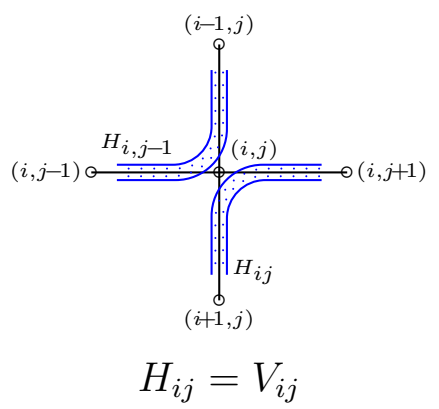

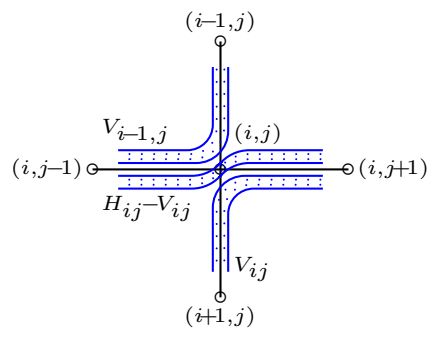

$H_{i j}>V_{i j}$

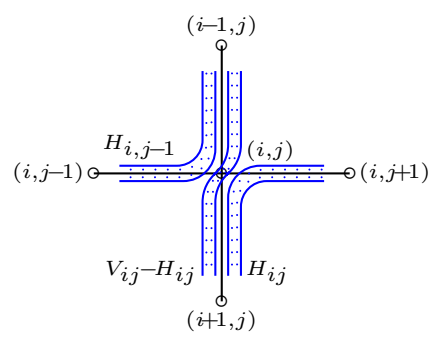

$V_{i j}>H_{i j}$

Figure 4: Path configurations through vertex $(i, j)$ for the cases of $(23)$. 
The three cases of (23) are shown diagrammatically in Figure 4, the path configurations which correspond to the vertex types of $\mathcal{V}(2)$ from Figure 3 are shown in Figure 5, and the path set of $\operatorname{LP}(5,2)$ which corresponds to the running example of (3), (11) and Figure 2 is shown in Figure 6. In order to assist in their visualization, some of the path segments in these diagrams have been shifted slightly away from the lattice edges on which they actually lie. Also, as indicated in the previous section, we are using matrix-type labeling of lattice points.

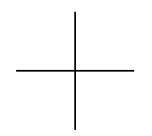

$(1)$

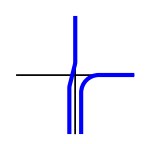

$(7)$

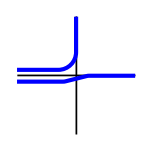

(13)

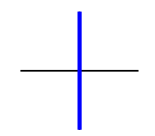

$(2)$

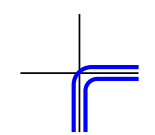

(8)

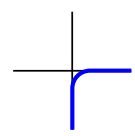

(3)

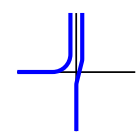

(9)

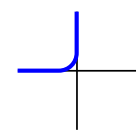

$(4)$

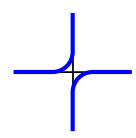

(10)

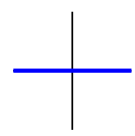

(5)

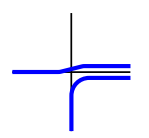

(11)

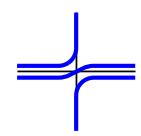

(18)

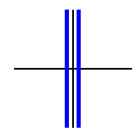

(6)

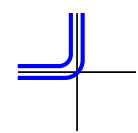

(12)

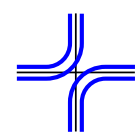

(19)

Figure 5: Path configurations for the 19 vertex types of $\mathcal{V}(2)$.

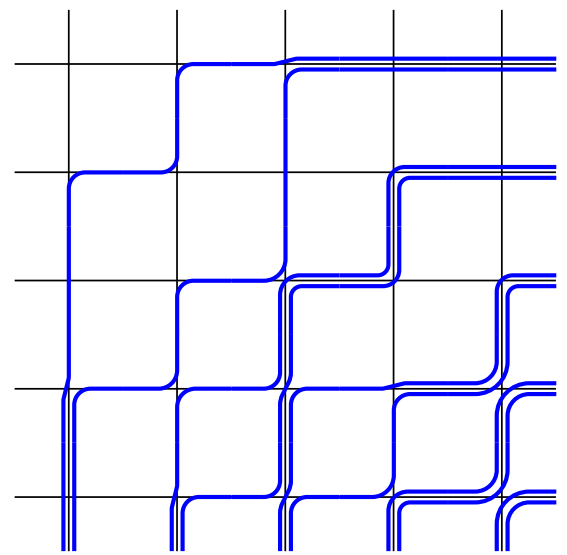

Figure 6: Set of lattice paths for the running example.

The case $\operatorname{LP}(n, 1)$ of path sets for standard alternating sign matrices is studied in detail in [9] as a particular case of osculating paths which start and end at fixed points on the lower and right boundaries of a rectangle. The correspondence between standard 
alternating sign matrices and such osculating paths is also considered in [14, Sec. 5], [15, Sec. 2], [31, Sec. 9] and [66, Sec. IV].

\section{Further Representations of Higher Spin Alternating Sign Matrices}

In this section, we describe three further combinatorial objects which are in bijection with higher spin alternating sign matrices: corner sum matrices, monotone triangles and complementary edge matrix pairs. These provide generalizations of previously-studied combinatorial objects in bijection with standard alternating sign matrices. We also describe certain path sets, namely fully packed loop configurations, which are closely related to complementary edge matrix pairs.

For $n \in \mathbb{P}$ and $r \in \mathbb{N}$, let the set of corner sum matrices be

$$
\begin{aligned}
\operatorname{CSM}(n, r):= & \left\{C=\left(\begin{array}{ccc}
C_{00} & \ldots & C_{0 n} \\
\vdots & & \vdots \\
C_{n 0} & \ldots & C_{n n}
\end{array}\right) \in \mathbb{N}^{(n+1) \times(n+1)} \mid\right. \\
& \bullet C_{0 k}=C_{k 0}=0, C_{k n}=C_{n k}=k r, \text { for all } k \in[n] \\
& \left.\bullet 0 \leq C_{i j}-C_{i, j-1} \leq r, \quad 0 \leq C_{i j}-C_{i-1, j} \leq r, \text { for all } i, j \in[n]\right\} .
\end{aligned}
$$

It can be checked that there is a bijection between $\operatorname{ASM}(n, r)$ and $\operatorname{CSM}(n, r)$ in which the corner sum matrix $C$ which corresponds to the higher spin alternating sign matrix $A$ is given by

$$
C_{i j}=\sum_{i^{\prime}=1}^{i} \sum_{j^{\prime}=1}^{j} A_{i^{\prime} j^{\prime}}, \text { for each } i, j \in[0, n]
$$

and inversely,

$$
A_{i j}=C_{i j}-C_{i, j-1}-C_{i-1, j}+C_{i-1, j-1}, \text { for each } i, j \in[n] \text {. }
$$

Combining the bijections $(8,9)$ between $\operatorname{EM}(n, r)$ and $\operatorname{ASM}(n, r)$, and $(25,26)$ between $\operatorname{ASM}(n, r)$ and $\operatorname{CSM}(n, r)$, the corner sum matrix $C$ which corresponds to the edge matrix pair $(H, V)$ is given by

$$
C_{i j}=\sum_{i^{\prime}=1}^{i} H_{i^{\prime} j}=\sum_{j^{\prime}=1}^{j} V_{i j^{\prime}}, \text { for each } i, j \in[0, n],
$$

and inversely,

$$
\begin{aligned}
H_{i j} & =C_{i j}-C_{i-1, j}, \text { for each } i \in[n], j \in[0, n] \\
V_{i j} & =C_{i j}-C_{i, j-1}, \text { for each } i \in[0, n], j \in[n] .
\end{aligned}
$$


The set $\operatorname{CSM}(n, 1)$ of corner sum matrices for standard alternating sign matrices was introduced in [59], and is also considered in [55].

The corner sum matrix which corresponds to the running example of (3) and (11) is

$$
\left(\begin{array}{lllllc}
0 & 0 & 0 & 0 & 0 & 0 \\
0 & 0 & 1 & 2 & 2 & 2 \\
0 & 1 & 1 & 2 & 4 & 4 \\
0 & 1 & 2 & 4 & 4 & 6 \\
0 & 2 & 3 & 5 & 6 & 8 \\
0 & 2 & 4 & 6 & 8 & 10
\end{array}\right) .
$$

Proceeding now to sets of monotone triangles, for $n \in \mathbb{P}$ and $r \in \mathbb{N}$, let $\operatorname{MT}(n, r)$ be the set of all triangular arrays $M$ of the form

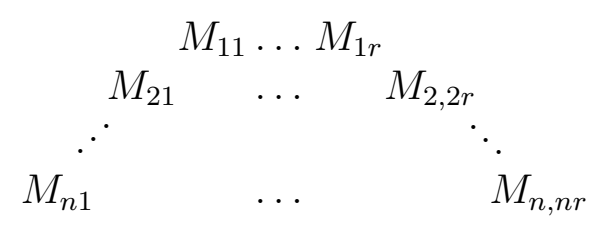

such that

- Each entry of $M$ is in $[n]$.

- In each row of $M$, any integer of $[n]$ appears at most $r$ times.

- $M_{i j} \leq M_{i, j+1}$ for each $i \in[n], j \in[i r-1]$.

- $M_{i+1, j} \leq M_{i j} \leq M_{i+1, j+r}$ for each $i \in[n-1], j \in[i r]$.

It follows that the last row of any monotone triangle in $\mathrm{MT}(n, r)$ consists of each integer of $[n]$ repeated $r$ times.

It can be checked that there is a bijection between $\operatorname{ASM}(n, r)$ and $\operatorname{MT}(n, r)$ in which the monotone triangle $M$ which corresponds to the higher spin alternating sign matrix $A$ is obtained by first using (8) to find the vertical edge matrix $V$ which corresponds to $A$, and then placing the integer $j V_{i j}$ times in row $i$ of $M$, for each $i, j \in[n]$, with these integers being placed in weakly increasing order along each row. (Note that there is alternative bijection in which the horizontal edge matrix $H$ which corresponds to $A$ is obtained, and the integer $i$ is then placed $H_{i j}$ times in row $j$ of $M$, for each $i, j \in[n]$.) For the inverse mapping, for each $i \in[0, n]$ and $j \in[n], V_{i j}$ is set to be the number of times that $j$ occurs in row $i$ of $M$, and $A$ is then obtained from $V$ using (9).

The set $\operatorname{MT}(n, 1)$ of monotone triangles for standard alternating sign matrices was introduced in [51], and is also studied in, for example, [34, 35, 52, 55, 70]. 
The monotone triangle which corresponds to the running example of (3) and (11) is

$$
\begin{array}{lllllllllll} 
& & & & & 2 & 3 & & & & \\
& & & & 1 & 3 & 4 & 4 & & & \\
& & & 1 & 2 & 3 & 3 & 5 & 5 & & \\
& & 1 & 1 & 2 & 3 & 3 & 4 & 5 & 5 & \\
& 1 & 1 & 2 & 2 & 3 & 3 & 4 & 4 & 5 & 5 .
\end{array}
$$

Proceeding finally to sets of complementary edge matrix pairs, for $n \in \mathbb{P}$ and $r \in \mathbb{N}$ we define

$$
\begin{aligned}
& \operatorname{CEM}(n, r):= \\
& \left\{(\bar{H}, \bar{V})=\left(\left(\begin{array}{ccc}
\bar{H}_{10} & \ldots & \bar{H}_{1 n} \\
\vdots & & \vdots \\
\bar{H}_{n 0} & \ldots & \bar{H}_{n n}
\end{array}\right),\left(\begin{array}{ccc}
\bar{V}_{01} & \ldots & \bar{V}_{0 n} \\
\vdots & & \vdots \\
\bar{V}_{n 1} & \ldots & \bar{V}_{n n}
\end{array}\right)\right) \in[0, r]^{n \times(n+1)} \times[0, r]^{(n+1) \times n} \mid\right. \\
& \text { - } \left.\bar{H}_{2 k-1,0}=\bar{H}_{n-2 k+2, n}=0, \quad \bar{V}_{0,2 k-1}=\bar{V}_{n, n-2 k+2}=r \text {, for all } k \in\left[\left\lceil\frac{n}{2}\right\rceil\right]\right) \\
& \text { - } \bar{H}_{2 k, 0}=\bar{H}_{n-2 k+1, n}=r, \quad \bar{V}_{0,2 k}=\bar{V}_{n, n-2 k+1}=0 \text {, for all } k \in\left[\left\lfloor\frac{n}{2}\right\rfloor\right] \\
& \text { - } \bar{V}_{i-1, j}+\bar{H}_{i, j-1}+\bar{V}_{i j}+\bar{H}_{i j}=2 r \text {, for all } i, j \in[n]
\end{aligned}
$$

It can be seen that there is a bijection between $\operatorname{EM}(n, r)$ (and hence $\operatorname{ASM}(n, r))$ and $\operatorname{CEM}(n, r)$ in which the complementary edge matrix pair $(\bar{H}, \bar{V})$ which corresponds to the edge matrix pair $(H, V)$ is given by

$$
\begin{aligned}
& \bar{H}_{i j}=\left\{\begin{array}{l}
H_{i j}, i+j \text { odd } \\
r-H_{i j}, i+j \text { even }
\end{array} \quad \text { for each } i \in[n], j \in[0, n]\right. \\
& \bar{V}_{i j}=\left\{\begin{array}{l}
r-V_{i j}, i+j \text { odd } \\
V_{i j}, i+j \text { even }
\end{array} \quad \text { for each } i \in[0, n], j \in[n] .\right.
\end{aligned}
$$

The complementary edge matrix pair which corresponds to the running example of (3) and (11) is

$$
(\bar{H}, \bar{V})=\left(\left(\begin{array}{llllll}
0 & 2 & 1 & 0 & 2 & 0 \\
2 & 1 & 2 & 0 & 0 & 2 \\
0 & 2 & 1 & 0 & 0 & 0 \\
2 & 1 & 1 & 1 & 0 & 2 \\
0 & 2 & 1 & 1 & 2 & 0
\end{array}\right),\left(\begin{array}{lllll}
2 & 0 & 2 & 0 & 2 \\
0 & 1 & 1 & 2 & 0 \\
1 & 0 & 1 & 2 & 2 \\
1 & 1 & 2 & 2 & 2 \\
0 & 1 & 0 & 1 & 0 \\
2 & 0 & 2 & 0 & 2
\end{array}\right)\right) .
$$

In analogy with the association of an edge matrix pair to a configuration of a statistical mechanical model, each entry of a complementary edge matrix pair can be assigned to an 
edge of the lattice, i.e., $\bar{H}_{i j}$ is assigned to the horizontal edge between $(i, j)$ and $(i, j+1)$, for each $i \in[n], j \in[0, n]$, and $\bar{V}_{i j}$ is assigned to the vertical edge between $(i, j)$ and $(i+1, j)$, for each $i \in[0, n], j \in[n]$. Also, in analogy with (12), we define the set of complementary vertex types as

$$
\overline{\mathcal{V}}(r):=\left\{\left(\bar{h}, \bar{v}, \bar{h}^{\prime}, \bar{v}^{\prime}\right) \in[0, r]^{4} \mid \bar{h}+\bar{v}+\bar{h}^{\prime}+\bar{v}^{\prime}=2 r\right\}
$$

so that the lattice point $(i, j)$ is associated with the complementary vertex type $\left(\bar{H}_{i, j-1}, \bar{V}_{i j}\right.$, $\left.\bar{H}_{i j}, \bar{V}_{i-1, j}\right) \in \overline{\mathcal{V}}(r)$, for each $i, j \in[n]$. Note that the mappings of each $\left(h, v, h^{\prime}, v^{\prime}\right) \in \mathcal{V}(r)$ to $\left(h, v, r-h^{\prime}, r-v^{\prime}\right)$, or of each $\left(h, v, h^{\prime}, v^{\prime}\right) \in \mathcal{V}(r)$ to $\left(r-h, r-v, h^{\prime}, v^{\prime}\right)$, give two bijections between $\mathcal{V}(r)$ and $\overline{\mathcal{V}}(r)$. The assignment of the entries of the complementary edge matrix pair of (33) to lattice edges is shown diagrammatically in Figure 7.

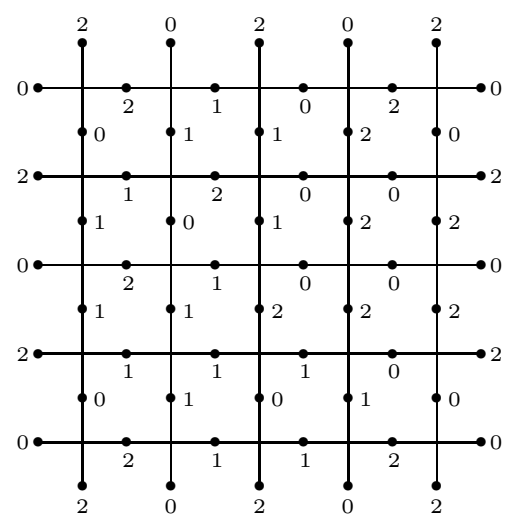

Figure 7: Assignment of entries of (33) to lattice edges.

We now define, for each $n \in \mathbb{P}$ and $r \in \mathbb{N}$, the set $\operatorname{FPL}(n, r)$ of fully packed loop configurations to be the set of all sets $P$ of nondirected open and closed lattice paths such that

- Successive points on each path of $P$ differ by $(-1,0),(1,0),(0,-1)$ or $(0,1)$.

- Each edge occupied by a path of $P$ is a horizontal edge between $(i, j)$ and $(i, j+1)$ with $i \in[0, n]$ and $j \in[n]$, or a vertical edge between $(i, j)$ and $(i+1, j)$ with $i \in[n]$ and $j \in[0, n]$.

- Any two edges occupied successively by a path of $P$ are different.

- Each edge is occupied by at most $r$ segments of paths of $P$.

- Each path of $P$ does not cross itself or any other path of $P$.

- Exactly $r$ segments of paths of $P$ pass through each (internal) point of $[n] \times[n]$.

- At each (external) point $(0,2 k-1)$ and $(n+1, n-2 k+2)$ for $k \in\left[\left\lceil\frac{n}{2}\right\rceil\right]$, and $(2 k, 0)$ and $(n-2 k+1, n+1)$ for $k \in\left[\left\lfloor\frac{n}{2}\right\rfloor\right]$, there are exactly $r$ endpoints of paths of $P$, these being the only lattice points which are path endpoints. 
Note that an open nondirected lattice path is a sequence $\left(p_{1}, \ldots, p_{m}\right)$ of points of $\mathbb{Z}^{2}$, for some $m \in \mathbb{P}$, where the reverse sequence $\left(p_{m}, \ldots, p_{1}\right)$ is regarded as the same path. The endpoints of such a path are $p_{1}$ and $p_{m}$, and the pairs of successive points are $p_{i}$ and $p_{i+1}$, for each $i \in[m-1]$. A closed nondirected lattice path is a sequence $\left(p_{1}, \ldots, p_{m}\right)$ of points of $\mathbb{Z}^{2}$, where reversal and all cyclic permutations of the sequence are regarded as the same path. Such a path has no endpoints, and its pairs of successive points are $p_{i}$ and $p_{i+1}$, for each $i \in[m-1]$, as well as $p_{1}$ and $p_{m}$. For the case of $P \in \operatorname{FPL}(n, r)$, a path of $P$ whose points are all internal, i.e., in $[n] \times[n]$, is closed, and a path of $P$ which has two external points, necessarily its endpoints, is open, even if the two external points are the same.

It can now be seen that there is a mapping from $\operatorname{FPL}(n, r)$ to $\operatorname{CEM}(n, r)$ in which the fully packed loop configuration $P$ is mapped to the complementary edge matrix pair $(\bar{H}, \bar{V})$ according to

$$
\begin{aligned}
\bar{H}_{i j}= & \text { number of segments of paths of } P \text { which occupy the edge between } \\
& (i, j) \text { and }(i, j+1), \text { for each } i \in[n], j \in[0, n] \\
\bar{V}_{i j}= & \text { number of segments of paths of } P \text { which occupy the edge between } \\
& (i+1, j) \text { and }(i, j), \text { for each } i \in[0, n], j \in[n] .
\end{aligned}
$$

A fully packed loop configuration of $\operatorname{FPL}(5,2)$ which maps to the complementary edge matrix pair of (33) is shown diagrammatically in Figure 8.

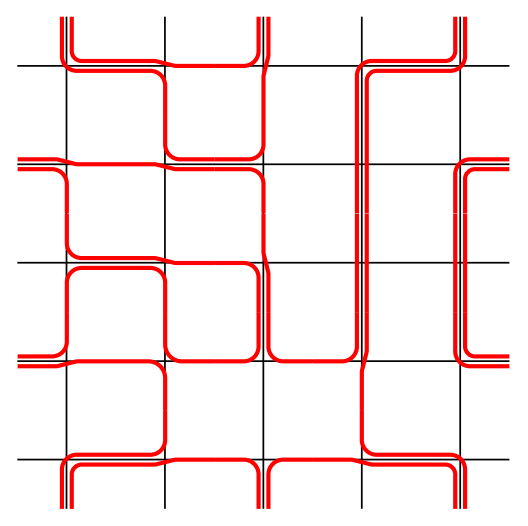

Figure 8: A fully packed loop configuration which maps to (33).

It can be checked that the mapping of (35) is surjective for each $r \in \mathbb{N}$ and $n \in \mathbb{P}$. Furthermore, for $r \in\{0,1\}$ or $n \in\{1,2\}$ it is injective, while for $r \geq 2$ and $n \geq 3$ it is not injective. This is due to the fact that if, for a complementary vertex type $\left(\bar{H}_{i, j-1}, \bar{V}_{i j}, \bar{H}_{i j}, \bar{V}_{i-1, j}\right) \in$ $\overline{\mathcal{V}}(r),(35)$ is used to assign appropriate numbers of path segments to the four edges surrounding the point $(i, j) \in[n] \times[n]$, then for $r \in\{0,1\}$ there is always a unique way to link 
these $2 r$ segments through $(i, j)$, whereas for $r \geq 2$ there can be several ways of linking the segments, such cases occurring for each $n \geq 3$. For example, for $r=2$ there is a unique way of linking the segments, except if $\left(\bar{H}_{i, j-1}, \bar{V}_{i j}, \bar{V}_{i-1, j}, \bar{H}_{i j}\right)=(1,1,1,1)$, in which case either of the configurations $\frac{f}{f}$ or $\_$can be used. Thus, since the example $(\bar{H}, \bar{V})$ of (33) and Figure 7 has the single case $(i, j)=(4,2)$ where this occurs, there are two fully packed loop configurations of $\operatorname{FPL}(5,2)$ which map to $(\bar{H}, \bar{V})$ : that of Figure 8 and that which differs from it by the configuration at $(4,2)$.

It follows that if each complementary vertex type $\left(\bar{h}, \bar{v}, \bar{h}^{\prime}, \bar{v}^{\prime}\right) \in \overline{\mathcal{V}}(r)$ is weighted by the number of ways of linking $2 r$ path segments corresponding to $\bar{h}, \bar{v}, \bar{h}^{\prime}$ and $\bar{v}^{\prime}$ through a vertex, then $|\operatorname{FPL}(n, r)|$ can be obtained as a weighted enumeration of $|\operatorname{ASM}(n, r)|$, in which each higher spin alternating sign matrix is weighted by the product of the weights of all the complementary vertex types associated with the corresponding complementary edge matrix pair.

The cases of $\operatorname{FPL}(n, 1)$, and of certain related sets which arise by imposing additional symmetry conditions, have been studied extensively. See for example [20, 21, 28, 29, 68, 75]. In these studies, each fully packed loop configuration is usually classified according to the link pattern formed among the external points by its open paths. This then leads to important results and conjectures, including unexpected connections with certain statistical mechanical models. See for example [24, 25] and references therein.

Link patterns related to certain higher spin integrable statistical mechanical models have been studied in [74]. Motivated by this work, it seems natural to define $\operatorname{FPL}(n, r)$ dis to be the set of fully packed loop configurations of $\operatorname{FPL}(n, r)$ for which each open path has distinct endpoints, and to define $\operatorname{FPL}(n, r)_{\text {adm }}$ to be the set of fully packed loop configurations of $\operatorname{FPL}(n, r)_{\text {dis }}$ for which the link pattern formed by the open paths is admissible, where admissibility of a link pattern is defined in [74, Sec. 2.5]. However, the mapping of (35) applied to either $\operatorname{FPL}(n, r)_{\text {dis }}$ or $\operatorname{FPL}(n, r)_{\text {adm }}$ still does not give a bijection to $\operatorname{CEM}(n, r)$ for $n \geq 3$ and $r \geq 2$. Some examples which show the failure of bijectivity in certain cases are provided in Figure 9: (a) and (b) are both in $\operatorname{FPL}(3,2)$ dis and map to the same element of $\operatorname{CEM}(3,2)$, showing that (35) is not injective between $\operatorname{FPL}(3,2)$ dis and $\operatorname{CEM}(3,2)$; (c) is not in $\operatorname{FPL}(3,2)$ adm and is the only element of $\operatorname{FPL}(3,2)$ which maps to its image in $\operatorname{CEM}(3,2)$ (since it does not contain the complementary vertex type $(1,1,1,1))$, showing that (35) is not surjective between $\operatorname{FPL}(3,2)$ adm and $\operatorname{CEM}(3,2)$; (d) is not in $\operatorname{FPL}(4,2)$ dis (since it contains an open path with both endpoints at $(2,0)$ ) and is the only element of $\operatorname{FPL}(4,2)$ which maps to its image in $\operatorname{CEM}(4,2)$, showing that $(35)$ is not surjective between $\operatorname{FPL}(4,2)_{\text {dis }}($ or $\operatorname{FPL}(4,2)$ adm $)$ and $\operatorname{CEM}(4,2)$. 


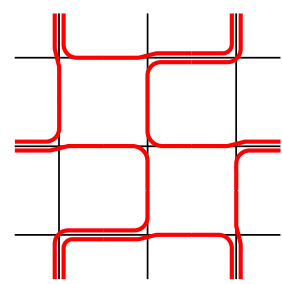

(a)

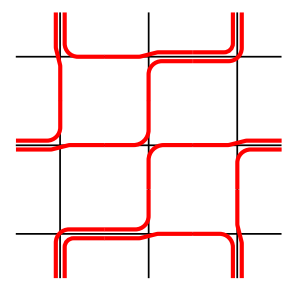

(b)

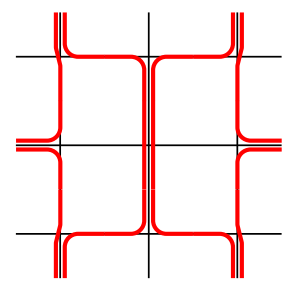

(c)

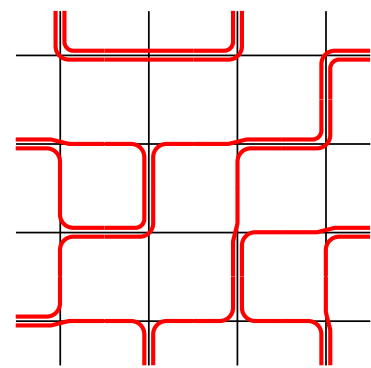

(d)

Figure 9: Further examples of fully packed loop configurations.

\section{The Alternating Sign Matrix Polytope}

In this section, we define the alternating sign matrix polytope in $\mathbb{R}^{n^{2}}$, using a halfspace description, and we show that its vertices are the standard alternating sign matrices of size $n$.

We begin by summarizing the facts about convex polytopes which will be needed here. For further information, see for example [73]. For $m \in \mathbb{P}$, a convex polytope in $\mathbb{R}^{m}$ can be defined as a bounded intersection of finitely-many closed affine halfspaces in $\mathbb{R}^{m}$, or equivalently as a convex hull of finitely-many points in $\mathbb{R}^{m}$. The equivalence of these descriptions is nontrivial and is proved, for example, in [73, Theorem 1.1]. It follows that hyperplanes in $\mathbb{R}^{m}$ can be included together with closed halfspaces in the first description, since a hyperplane is simply the intersection of the two closed halfspaces which meet at the hyperplane. The dimension, $\operatorname{dim} \mathcal{P}$, of a convex polytope $\mathcal{P}$ is defined to be the dimension of its affine hull, aff $(\mathcal{P}):=\left\{\lambda p_{1}+(1-\lambda) p_{2} \mid p_{1}, p_{2} \in \mathcal{P}, \lambda \in \mathbb{R}\right\}$. A face of $\mathcal{P}$ is an intersection of $\mathcal{P}$ with any hyperplane for which $\mathcal{P}$ is a subset of one of the two closed halfspaces determined by the hyperplane. If a face contains only one point, that point is known as a vertex. Thus, the set of vertices, vert $\mathcal{P}$, of $\mathcal{P} \subset \mathbb{R}^{m}$ is the set of points $p \in \mathcal{P}$ for which there exists a closed affine halfspace $\mathcal{S}$ in $\mathbb{R}^{m}$ such that $\mathcal{P} \cap \mathcal{S}=\{p\}$. It can be shown that vert $\mathcal{P}$ is also the set of points $p \in \mathcal{P}$ which do not lie in the interior of any line segment in $\mathcal{P}$, i.e., $p \in \mathcal{P}$ is a vertex of $\mathcal{P}$ if and only if there do not exist $\lambda \in(0,1)_{\mathbb{R}}$ and $p_{1} \neq p_{2} \in \mathcal{P}$ with $p=\lambda p_{1}+(1-\lambda) p_{2}$. Any convex polytope $\mathcal{P}$ has only finitely-many vertices, and is the convex hull of these vertices, or equivalently the set of all convex combinations of its vertices, $\mathcal{P}=\left\{\sum_{p \in \operatorname{vert} \mathcal{P}} \lambda_{p} p \mid \lambda_{p} \in[0,1]_{\mathbb{R}}\right.$ for each $\left.p \in \operatorname{vert} \mathcal{P}, \sum_{p \in \operatorname{vert} \mathcal{P}} \lambda_{p}=1\right\}$. Convex polytopes $\mathcal{P} \subset \mathbb{R}^{m}$ and $\mathcal{P}^{\prime} \subset \mathbb{R}^{m^{\prime}}$ are defined to be affinely isomorphic if there is an affine map $\phi: \mathbb{R}^{m} \rightarrow \mathbb{R}^{m^{\prime}}$ which is bijective between $\mathcal{P}$ and $\mathcal{P}^{\prime}$. In such cases, vert $\mathcal{P}^{\prime}=\phi(\operatorname{vert} \mathcal{P})$. Finally, a convex polytope whose vertices all have integer coordinates is known as an integral polytope or a lattice polytope. 
We now define, for $n \in \mathbb{P}$,

$$
\begin{aligned}
& \mathcal{A}_{n}:= \\
& \left\{x=\left(\begin{array}{c|c}
x_{11} \ldots & x_{1 n} \\
\vdots & \vdots \\
x_{n 1} \ldots & x_{n n}
\end{array}\right) \in \mathbb{R}^{n \times n} \mid \begin{array}{l}
\bullet \sum_{j^{\prime}=1}^{n} x_{i j^{\prime}}=\sum_{i^{\prime}=1}^{n} x_{i^{\prime} j}=1 \text { for all } i, j \in[n] \\
\bullet \sum_{j^{\prime}=1}^{j} x_{i j^{\prime}} \geq 0 \text { for all } i \in[n], j \in[n-1] \\
\bullet \sum_{j^{\prime}=j}^{n} x_{i j^{\prime}} \geq 0 \text { for all } i \in[n], j \in[2, n] \\
\bullet \sum_{i^{\prime}=1}^{i} x_{i^{\prime} j} \geq 0 \text { for all } i \in[n-1], j \in[n] \\
\bullet \sum_{i^{\prime}=i}^{n} x_{i^{\prime} j} \geq 0 \text { for all } i \in[2, n], j \in[n]
\end{array}\right\} \\
& =\left\{x=\left(\begin{array}{c|cc}
x_{11} \ldots & x_{1 n} \\
\vdots & & \vdots \\
x_{n 1} & \ldots & x_{n n}
\end{array}\right) \in \mathbb{R}^{n \times n} \mid \begin{array}{l}
\bullet \sum_{j^{\prime}=1}^{n} x_{i j^{\prime}}=\sum_{i^{\prime}=1}^{n} x_{i^{\prime} j}=1 \text { for all } i, j \in[n] \\
\bullet 0 \leq \sum_{j^{\prime}=1}^{j} x_{i j^{\prime}} \leq 1 \text { for all } i \in[n], j \in[n-1] \\
\bullet 0 \leq \sum_{i^{\prime}=1}^{i} x_{i^{\prime} j} \leq 1 \text { for all } i \in[n-1], j \in[n]
\end{array}\right\} .
\end{aligned}
$$

In other words, $\mathcal{A}_{n}$ is the set of $n \times n$ real-entry matrices for which all complete row and column sums are 1 , and all partial row and column sums extending from each end of the row or column are nonnegative.

It can be seen that each entry of any matrix of $\mathcal{A}_{n}$ is between -1 and 1 , and that if the entry is in the first or last row or column, then it is between 0 and 1 , so that $\mathcal{A}_{n}$ is a bounded subset of $\mathbb{R}^{n^{2}}$. Since $\mathcal{A}_{n}$ is also an intersection of finitely-many closed halfspaces and hyperplanes in $\mathbb{R}^{n^{2}}$, it is a convex polytope in $\mathbb{R}^{n^{2}}$, and will be referred to as the alternating sign matrix polytope. This polytope was defined independently, using a convex hull description, in [65].

An example of an element of $\mathcal{A}_{4}$ is

$$
x=\left(\begin{array}{cccc}
.3 & 0 & .6 & .1 \\
.2 & .5 & -.6 & .9 \\
.5 & -.5 & 1 & 0 \\
0 & 1 & 0 & 0
\end{array}\right)
$$

Defining

$$
\mathcal{B}_{n}:=\left\{x \in \mathcal{A}_{n} \mid x_{i j} \geq 0 \text { for each } i, j \in[n]\right\},
$$

it can be seen that this is the set of doubly stochastic matrices of size $n$, i.e., nonnegative real-entry $n \times n$ matrices for which all complete row and column sums are 1 . This is the convex polytope in $\mathbb{R}^{n^{2}}$ often known as the Birkhoff polytope. See for example [73, Ex. 0.12] and references therein.

It now follows that the higher spin alternating sign matrices and semimagic squares of 
size $n$ with line sum $r$ are the integer points of the $r$-th dilates of $\mathcal{A}_{n}$ and $\mathcal{B}_{n}$ respectively,

$$
\operatorname{ASM}(n, r)=r \mathcal{A}_{n} \cap \mathbb{Z}^{n^{2}}, \quad \operatorname{SMS}(n, r)=r \mathcal{B}_{n} \cap \mathbb{N}^{n^{2}},
$$

where the $r$-th dilate of a set $P \subset \mathbb{R}^{m}$ is simply $r P:=\{r x \mid x \in P\}$.

It also follows that aff $\mathcal{A}_{n}=\operatorname{aff}_{n}=\left\{x \in \mathbb{R}^{n \times n} \mid \sum_{j^{\prime}=1}^{n} x_{i j^{\prime}}=\sum_{i^{\prime}=1}^{n} x_{i^{\prime} j}=1\right.$ for all $i, j \in$ $[n]\}$, and that of the $2 n$ linear equations in $n^{2}$ variables within this set, only $2 n-1$ equations are independent, so that

$$
\operatorname{dim} \mathcal{A}_{n}=\operatorname{dim} \mathcal{B}_{n}=(n-1)^{2} .
$$

This is effectively equivalent to the fact that any $x \in \operatorname{aff} \mathcal{A}_{n}=\operatorname{aff} \mathcal{B}_{n}$ can be obtained by freely choosing the entries of any $(n-1) \times(n-1)$ submatrix of $x$, the remaining $2 n-1$ entries of $x$ then being determined by the condition that each row and column sum is 1 .

We also define, for $n \in \mathbb{P}$,

$$
\begin{gathered}
\mathcal{E}_{n}:=\left\{(h, v)=\left(\left(\begin{array}{ccc}
h_{10} & \ldots & h_{1 n} \\
\vdots & & \vdots \\
h_{n 0} & \ldots & h_{n n}
\end{array}\right),\left(\begin{array}{ccc}
v_{01} & \ldots & v_{0 n} \\
\vdots & & \vdots \\
v_{n 1} & \ldots & v_{n n}
\end{array}\right)\right) \in[0,1]_{\mathbb{R}}^{n \times(n+1)} \times[0,1]_{\mathbb{R}}^{(n+1) \times n} \mid\right. \\
\left.h_{i 0}=v_{0 j}=0, h_{i n}=v_{n j}=1, h_{i, j-1}+v_{i j}=v_{i-1, j}+h_{i j}, \text { for all } i, j \in[n]\right\} .
\end{gathered}
$$

This is a convex polytope in $\mathbb{R}^{2 n(n+1)}$, which we shall refer to as the edge matrix polytope. It can be seen that $\operatorname{EM}(n, r)=r \mathcal{E}_{n} \cap \mathbb{Z}^{2 n(n+1)}$, and that there is a bijection between $\mathcal{A}_{n}$ and $\mathcal{E}_{n}$ in which the $(h, v) \in \mathcal{E}_{n}$ which corresponds to $x \in \mathcal{A}_{n}$ is given by

$$
\begin{aligned}
& h_{i j}=\sum_{j^{\prime}=1}^{j} x_{i j^{\prime}}, \quad \text { for each } i \in[n], j \in[0, n] \\
& v_{i j}=\sum_{i^{\prime}=1}^{i} x_{i^{\prime} j}, \quad \text { for each } i \in[0, n], j \in[n],
\end{aligned}
$$

and inversely,

$$
x_{i j}=h_{i j}-h_{i, j-1} \quad \text { or } \quad x_{i j}=v_{i j}-v_{i-1, j}, \quad \text { for each } i, j \in[n] .
$$

Furthermore, (42) can be regarded as a linear map from $\mathbb{R}^{n^{2}}$ to $\mathbb{R}^{2 n(n+1)}$, and each of the equations of (43) can be regarded as a linear map from $\mathbb{R}^{2 n(n+1)}$ to $\mathbb{R}^{n^{2}}$, implying that $\mathcal{A}_{n}$ and $\mathcal{E}_{n}$ are affinely isomorphic. These mappings, when restricted to $\operatorname{ASM}(n, r)$ and $\operatorname{EM}(n, r)$, are simply the mappings (8) and (9).

It follows that, similarly to (10),

$$
\sum_{i, j=1}^{n} h_{i j}=\sum_{i, j=1}^{n} v_{i j}=n(n+1) / 2 \text { for each }(h, v) \in \mathcal{E}_{n} .
$$


It is shown in $[10,67]$ that the vertices of the Birkhoff polytope $\mathcal{B}_{n}$ are the permutation matrices of size $n$, so that $\mathcal{B}_{n}$ is an integral convex polytope. We now state and prove the corresponding result for $\mathcal{A}_{n}$. This result was obtained independently in [65].

Theorem 1. The vertices of the alternating sign matrix polytope $\mathcal{A}_{n}$ are the standard alternating sign matrices of size $n$.

Proof. We shall show that the vertices of the edge matrix polytope $\mathcal{E}_{n}$ are the edge matrix pairs of $\operatorname{EM}(n, 1)$, i.e., $\operatorname{vert} \mathcal{E}_{n}=\operatorname{EM}(n, 1)$. It then follows, since $\mathcal{E}_{n}$ and $\mathcal{A}_{n}$ are affinely isomorphic with mapping (43), and since (43) maps $\operatorname{EM}(n, 1)$ to $\operatorname{ASM}(n, 1)$, that $\operatorname{vert} \mathcal{A}_{n}=\operatorname{ASM}(n, 1)$ as required.

We first show that $\operatorname{EM}(n, 1) \subset \operatorname{vert} \mathcal{E}_{n}$. Consider any $(H, V) \in \operatorname{EM}(n, 1)$. From $(7)$, this is a pair of matrices with a total of $2 n(n+1)\{0,1\}$-entries, of which, due to $(10), n(n+1)$ are 0's and $n(n+1)$ are 1's. Now define the halfspace $\mathcal{S}=\left\{(y, z) \in \mathbb{R}^{n \times(n+1)} \times \mathbb{R}^{(n+1) \times n}\right.$ $\left.\sum_{i, j=1}^{n}\left(H_{i j} y_{i j}+V_{i j} z_{i j}\right) \geq n(n+1)\right\}$, and consider any matrix pair $(h, v) \in \mathcal{E}_{n} \cap \mathcal{S}$. Due to $(7),(41)$ and (44), one such matrix pair is $(H, V)$. Also, $(h, v) \in \mathcal{E}_{n}$ implies, using (41) and (44), that each of the $2 n(n+1)$ entries of $(h, v)$ is between 0 and 1 inclusive, and that they all sum to $n(n+1)$, while $(h, v) \in \mathcal{S}$ implies that the $n(n+1)$ entries of $(h, v)$ in the same positions as the 1 's of $(H, V)$ sum to at least $n(n+1)$. It can be seen that these conditions are only satisfied if $(h, v)=(H, V)$. Therefore, $\mathcal{E}_{n} \cap \mathcal{S}=\{(H, V)\}$, implying that $(H, V) \in \operatorname{vert} \mathcal{E}_{n}$ as required. (Note that alternatively it could have been shown here that $\mathcal{E}_{n} \cap\left\{(y, z) \in \mathbb{R}^{n \times(n+1)} \times \mathbb{R}^{(n+1) \times n} \mid \sum_{i, j=1}^{n} H_{i j} y_{i j} \geq n(n+1) / 2\right\}=\{(H, V)\}$ or that $\left.\mathcal{E}_{n} \cap\left\{(y, z) \in \mathbb{R}^{n \times(n+1)} \times \mathbb{R}^{(n+1) \times n} \mid \sum_{i, j=1}^{n} V_{i j} z_{i j} \geq n(n+1) / 2\right\}=\{(H, V)\}.\right)$

We now show that $\operatorname{vert} \mathcal{E}_{n} \subset \operatorname{EM}(n, 1)$. Consider any $(h, v) \in \mathcal{E}_{n} \backslash \operatorname{EM}(n, 1)$. We shall eventually deduce that $(h, v) \notin \operatorname{vert} \mathcal{E}_{n}$, which gives the required result. Similarly to the association of edge matrix pairs with configurations of a statistical mechanical model, we associate $h_{i j}$ with the horizontal edge between lattice points $(i, j)$ and $(i, j+1)$, for each $i \in[n], j \in[0, n]$, and $v_{i j}$ with the vertical edge between lattice points $(i, j)$ and $(i+1, j)$, for each $i \in[0, n], j \in[n]$ (using matrix-type labeling of lattice points). Since $\operatorname{EM}(n, 1)=\mathcal{E}_{n} \cap \mathbb{Z}^{2 n(n+1)},(h, v) \notin \operatorname{EM}(n, 1)$ implies that at least one entry of $(h, v)$ is nonintegral (or in fact in $(0,1)_{\mathbb{R}}$ ). Now, from $(41),(h, v) \in \mathcal{E}_{n}$ implies that $h_{i 0}=v_{0 j}=0$ and $h_{i n}=v_{n j}=1$, for each $i, j \in[n]$, so that any nonintegral entry of $(h, v)$ must be associated with one of the $2 n(n-1)$ internal edges, (i.e., the horizontal edges between $(i, j)$ and $(i, j+1)$, for $i \in[n], j \in[n-1]$, and the vertical edges between $(i, j)$ and $(i+1, j)$, for each $i \in[n-1], j \in[n])$. Also from $(41),(h, v) \in \mathcal{E}_{n}$ implies that

$$
h_{i, j-1}+v_{i j}=v_{i-1, j}+h_{i j}
$$


for each $i, j \in[n]$. But if any one of the four entries in (45) is nonintegral, then at least one of the others must also be nonintegral. Therefore, the existence among the entries of $(h, v)$ of a noninteger implies the existence of two further nonintegers, among each of the other three entries of the two cases of (45) in which the initial nonintegral entry appears. It now follows by repeatedly applying this argument, and since the internal edges form a finite and closed grid, that there exists at least one cycle of internal edges associated with noninteger entries of $(h, v)$.

We select any such cycle, give it an orientation, say anticlockwise, and denote the sets of points $(i, j)$ for which the horizontal edge between $(i, j)$ and $(i, j+1)$ is in the cycle and directed right or left as respectively $\mathcal{H}_{+}$or $\mathcal{H}_{-}$, and the sets of points $(i, j)$ for which the vertical edge between $(i, j)$ and $(i+1, j)$ is in the cycle and directed up or down as respectively $\mathcal{V}_{+}$or $\mathcal{V}_{-}$. An example of such a cycle, for the $(h, v) \in \mathcal{E}_{4}$ which corresponds to the example $x \in \mathcal{A}_{4}$ of (37) is shown diagrammatically in Figure 10. For this example,

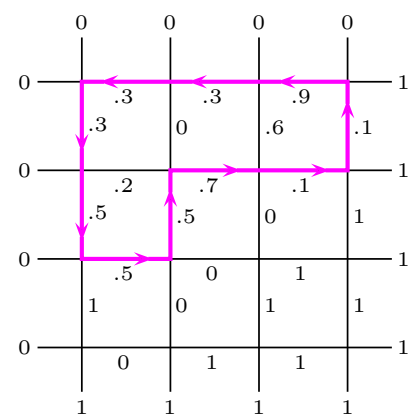

Figure 10: A cycle of nonintegers for the example of (37).

$\mathcal{H}_{+}=\{(2,2),(2,3),(3,1)\}, \mathcal{H}_{-}=\{(1,1),(1,2),(1,3)\}, \mathcal{V}_{+}=\{(1,4),(2,2)\}$ and $\mathcal{V}_{-}=$ $\{(1,1),(2,1)\}$. We now define, for $\mu \in \mathbb{R}$, a matrix pair $\left(h^{\prime}(\mu), v^{\prime}(\mu)\right)$, with entries $h^{\prime}(\mu)_{i j}$ for $i \in[n], j \in[0, n]$, and $v^{\prime}(\mu)_{i j}$ for $i \in[0, n], j \in[n]$, given by

$$
h^{\prime}(\mu)_{i j}=\left\{\begin{array}{l}
h_{i j}+\mu,(i, j) \in \mathcal{H}_{+} \\
h_{i j}-\mu,(i, j) \in \mathcal{H}_{-} \\
h_{i j}, \text { otherwise }
\end{array} \quad v^{\prime}(\mu)_{i j}=\left\{\begin{array}{l}
v_{i j}+\mu,(i, j) \in \mathcal{V}_{+} \\
v_{i j}-\mu,(i, j) \in \mathcal{V}_{-} \\
v_{i j}, \text { otherwise }
\end{array}\right.\right.
$$

For the example of Figure 10,

$$
\left(h^{\prime}(\mu), v^{\prime}(\mu)\right)=\left(\left(\begin{array}{ccccc}
0 & .3-\mu & .3-\mu & .9-\mu & 1 \\
0 & .2 & .7+\mu & .1+\mu & 1 \\
0 & .5+\mu & 0 & 1 & 1 \\
0 & 0 & 1 & 1 & 1
\end{array}\right),\left(\begin{array}{cccc}
0 & 0 & 0 & 0 \\
.3-\mu & 0 & .6 & .1+\mu \\
.5-\mu & .5+\mu & 0 & 1 \\
1 & 0 & 1 & 1 \\
1 & 1 & 1 & 1
\end{array}\right)\right)
$$

We now check whether $\left(h^{\prime}(\mu), v^{\prime}(\mu)\right) \in \mathcal{E}_{n}$. By using (46) to replace each entry of $(h, v)$ in (45) with an entry of $\left(h^{\prime}(\mu), v^{\prime}(\mu)\right)$, it can be checked that the equation $h^{\prime}(\mu)_{i, j-1}+$ 
$v^{\prime}(\mu)_{i j}=v^{\prime}(\mu)_{i-1, j}+h^{\prime}(\mu)_{i j}$ is satisfied for each $i, j \in[n]$, since if the cycle does not pass through $(i, j)$, then the required equation is immediately obtained, while for all possible configurations (of which there are six), and both possible directions, in which the cycle can pass through $(i, j)$, all explicit appearances of $\mu$ cancel out, again leaving the required equation. The conditions $h^{\prime}(\mu)_{i 0}=v^{\prime}(\mu)_{0 j}=0$ and $h^{\prime}(\mu)_{i n}=v^{\prime}(\mu)_{n j}=1$ are also met for each $i, j \in[n]$, since $\left(h^{\prime}(\mu), v^{\prime}(\mu)\right)$ and $(h, v)$ match for these entries. It only remains for the conditions $h^{\prime}(\mu)_{i j}, v^{\prime}(\mu)_{i j} \in[0,1]_{\mathbb{R}}$ to be checked for each $i, j \in[n]$, and it can be seen that these are satisfied provided that $-\mu_{-} \leq \mu \leq \mu_{+}$, where

$$
\begin{gathered}
\mu_{-}:=\min \left(\left\{h_{i j} \mid(i, j) \in \mathcal{H}_{+}\right\} \cup\left\{v_{i j} \mid(i, j) \in \mathcal{V}_{+}\right\}\right. \\
\left.\cup\left\{1-h_{i j} \mid(i, j) \in \mathcal{H}_{-}\right\} \cup\left\{1-v_{i j} \mid(i, j) \in \mathcal{V}_{-}\right\}\right) \\
\mu_{+}:=\min \left(\left\{1-h_{i j} \mid(i, j) \in \mathcal{H}_{+}\right\} \cup\left\{1-v_{i j} \mid(i, j) \in \mathcal{V}_{+}\right\}\right. \\
\left.\cup\left\{h_{i j} \mid(i, j) \in \mathcal{H}_{-}\right\} \cup\left\{v_{i j} \mid(i, j) \in \mathcal{V}_{-}\right\}\right) .
\end{gathered}
$$

The facts that $h_{i j} \in(0,1)_{\mathbb{R}}$ for each $(i, j) \in \mathcal{H}_{ \pm}$, and $v_{i j} \in(0,1)_{\mathbb{R}}$ for each $(i, j) \in \mathcal{V}_{ \pm}$, imply that $\mu_{-}$and $\mu_{+}$are both positive, so that there exists a finite-length, closed interval of values of $\mu$ for which $\left(h^{\prime}(\mu), v^{\prime}(\mu)\right) \in \mathcal{E}_{n}$. For the example of Figure $10, \mu_{-}=.1$ and $\mu_{+}=.3$. Finally, we define $\left(h_{ \pm}, v_{ \pm}\right):=\left(h^{\prime}\left( \pm \mu_{ \pm}\right), v^{\prime}\left( \pm \mu_{ \pm}\right)\right)$, so that it follows that $\left(h_{ \pm}, v_{ \pm}\right) \in \mathcal{E}_{n},\left(h_{-}, v_{-}\right) \neq\left(h_{+}, v_{+}\right)$, and

$$
(h, v)=\frac{\mu_{+}}{\mu_{-}+\mu_{+}}\left(h_{-}, v_{-}\right)+\frac{\mu_{-}}{\mu_{-}+\mu_{+}}\left(h_{+}, v_{+}\right) .
$$

Therefore, $(h, v)$ lies in the interior of a line segment between two points of $\mathcal{E}_{n}$, and so, as required, $(h, v) \notin \operatorname{vert} \mathcal{E}_{n}$. This concludes the proof of Theorem 1.

It follows immediately from Theorem 1 that the alternating sign matrix polytope and edge matrix polytope are integral (and that the edge matrix polytope is $0 / 1$, i.e., a polytope each of whose vertex coordinates is 0 or 1 ), a fact which will be important in the enumeration of higher spin alternating sign matrices in the next section.

It also follows from Theorem 1 that $\mathcal{A}_{n}$ can be described as the convex hull of the alternating sign matrices of size $n$, or equivalently that

$$
\begin{aligned}
& \mathcal{A}_{n}= \\
& \left.\qquad \sum_{A \in \operatorname{ASM}(n, 1)} \lambda_{A} A \mid \lambda_{A} \in[0,1]_{\mathbb{R}} \text { for each } A \in \operatorname{ASM}(n, 1), \sum_{A \in \operatorname{ASM}(n, 1)} \lambda_{A}=1\right\} .
\end{aligned}
$$

The way in which this conclusion has been reached here depends on the general theorem that any convex polytope has both a halfspace and convex hull description. However, (50) can also be derived more directly. It follows immediately from (1) and (36) that the RHS of (50) is a subset of the LHS, i.e., that every convex combination of standard alternating 
sign matrices of size $n$ is an element of $\mathcal{A}_{n}$. Conversely, every element of $\mathcal{A}_{n}$ is a convex combination of elements of $\operatorname{ASM}(n, 1)$ due to the following argument, which we owe to [65]. For each $x \in \mathcal{A}_{n}$, let $\nu(x)$ be the number of nonintegers among the entries of $(h, v)$, where $(h, v) \in \mathcal{E}_{n}$ corresponds to $x$ by (42). Now consider the following recursive process applied to $x \in \mathcal{A}_{n}$. If $\nu(x)=0$, then $x \in \operatorname{ASM}(n, 1)$, and the process is terminated. If $\nu(x)$ is positive, then let $x_{ \pm} \in \mathcal{A}_{n}$ correspond to $\left(h_{ \pm}, v_{ \pm}\right) \in \mathcal{E}_{n}$, where $\left(h_{ \pm}, v_{ \pm}\right)$are defined in terms of $(h, v)$ as in the proof of Theorem 1. Using (49), write $x$ as the convex combination $x=\frac{\mu_{+}}{\mu_{-}+\mu_{+}} x_{-}+\frac{\mu_{-}}{\mu_{-}+\mu_{+}} x_{+}$, where it can be seen that $\nu\left(x_{ \pm}\right)<\nu(x)$. Now reapply the process to $x_{-}$and $x_{+}$. It follows that any $x \in \mathcal{A}_{n}$ can thus be completely decomposed as a convex combination of elements of $\operatorname{ASM}(n, 1)$.

Faces of the alternating sign polytope other than those given by its vertices are studied in detail in [65], but we shall not consider these here.

\section{Enumeration of Higher Spin Alternating Sign Matrices of Fixed Size}

In this section, we use the general theory of the enumeration of integer points in integer dilates of integral convex polytopes to obtain results on the enumeration of higher spin alternating sign matrices of fixed size.

We begin by summarizing the components of this theory which will be needed here. For further information, more general results, and references, see for example [7] or [63, Sec. 4.6]. For an integral convex polytope $\mathcal{P}$ in $\mathbb{R}^{m}$ with relative interior $\mathcal{P}^{\circ}$, there exists a unique function $\mathcal{L}_{\mathcal{P}}(r)$, where $r$ can be regarded as a complex variable, with the properties that:

(i) $\mathcal{L}_{\mathcal{P}}(r)$ is a polynomial in $r$ of degree $\operatorname{dim} \mathcal{P}$.

(ii) $\left|r \mathcal{P} \cap \mathbb{Z}^{m}\right|=\mathcal{L}_{\mathcal{P}}(r)$, for each $r \in \mathbb{N}$.

(iii) $\left|r \mathcal{P}^{\circ} \cap \mathbb{Z}^{m}\right|=(-1)^{\operatorname{dim} \mathcal{P}} \mathcal{L}_{\mathcal{P}}(-r)$, for each $r \in \mathbb{N}$.

(iv) $\mathcal{L}_{\mathcal{P}}(r)$ can be expressed in the form $\mathcal{L}_{\mathcal{P}}(r)=\sum_{k=0}^{\operatorname{dim} \mathcal{P}} c_{k}\left(\begin{array}{c}r+k \\ \operatorname{dim} \mathcal{P}\end{array}\right)$, with $c_{k} \in \mathbb{N}$ for each $k \in[0, \operatorname{dim} \mathcal{P}]$.

The function $\mathcal{L}_{\mathcal{P}}(r)$ is known as the Ehrhart polynomial of $\mathcal{P}$. It can be seen that $\mathcal{L}_{\mathcal{P}}(0)=$ $c_{\operatorname{dim} \mathcal{P}}=1$, and that the Ehrhart polynomial can be obtained explicitly by finding $\mathcal{L}_{\mathcal{P}}(r)$ for $\operatorname{dim} \mathcal{P}$ further integers $r$ by directly enumerating the number of lattice points $\left|r \mathcal{P} \cap \mathbb{Z}^{m}\right|$ for positive $r$, or $\left|-r \mathcal{P}^{\circ} \cap \mathbb{Z}^{m}\right|$ for negative $r$, and then interpolating. 
For the alternating sign matrix polytope $\mathcal{A}_{n}$, the relative interior $\mathcal{A}_{n}^{\circ}$ is obtained by simply replacing each weak inequality in (36) by a strict inequality. Defining, for $n \in \mathbb{P}$ and $r \in \mathbb{N}$,

$$
\operatorname{ASM}^{\circ}(n, r):=r \mathcal{A}_{n}^{\circ} \cap \mathbb{Z}^{n^{2}}
$$

it is seen that

$$
\begin{aligned}
& \operatorname{ASM}^{\circ}(n, r):= \\
& \left.\left\{\begin{array}{c|cc}
A_{11} \ldots & A_{1 n} \\
\vdots & & \vdots \\
A_{n 1} \ldots & A_{n n}
\end{array}\right) \in \mathbb{Z}^{n \times n} \mid \begin{array}{l}
\bullet \sum_{j^{\prime}=1}^{n} A_{i j^{\prime}}=\sum_{i^{\prime}=1}^{n} A_{i^{\prime} j}=r \text { for all } i, j \in[n] \\
\bullet \sum_{j^{\prime}=1}^{j} A_{i j^{\prime}} \geq 1 \text { for all } i \in[n], j \in[n-1] \\
\bullet \sum_{j^{\prime}=j}^{n} A_{i j^{\prime}} \geq 1 \text { for all } i \in[n], j \in[2, n] \\
\bullet \sum_{i^{\prime}=1}^{i} A_{i^{\prime} j} \geq 1 \text { for all } i \in[n-1], j \in[n] \\
\bullet \sum_{i^{\prime}=i}^{n} A_{i^{\prime} j} \geq 1 \text { for all } i \in[2, n], j \in[n]
\end{array}\right\} \\
& \left.=\left\{\begin{array}{c|cc}
A_{11} \ldots & A_{1 n} \\
\vdots & & \vdots \\
A_{n 1} \ldots & A_{n n}
\end{array}\right) \in \mathbb{Z}^{n \times n} \mid \begin{array}{l}
\bullet \sum_{j^{\prime}=1}^{n} A_{i j^{\prime}}=\sum_{i^{\prime}=1}^{n} A_{i^{\prime} j}=r \text { for all } i, j \in[n] \\
\bullet 1 \leq \sum_{j^{\prime}=1}^{j} A_{i j^{\prime}} \leq r-1 \text { for all } i \in[n], j \in[n-1] \\
\bullet 1 \leq \sum_{i^{\prime}=1}^{i} A_{i^{\prime} j} \leq r-1 \text { for all } i \in[n-1], j \in[n]
\end{array}\right\} .
\end{aligned}
$$

Thus, $\operatorname{ASM}^{\circ}(n, r)$ is the set of $n \times n$ integer-entry matrices for which all complete row and column sums are $r$, and all partial row and column sums extending from each end of the row or column are positive. It follows that

$$
\left\{\left(\begin{array}{ccc}
A_{11}+1 & \ldots & A_{1 n}+1 \\
\vdots & & \vdots \\
A_{n 1}+1 & \ldots & A_{n n}+1
\end{array}\right) \mid\left(\begin{array}{ccc}
A_{11} & \ldots & A_{1 n} \\
\vdots & & \vdots \\
A_{n 1} & \ldots & A_{n n}
\end{array}\right) \in \operatorname{ASM}(n, r-n)\right\} \subset \operatorname{ASM}^{\circ}(n, r)
$$

for each $n, r \in \mathbb{P}$ with $r \geq n$,

and that

$$
\operatorname{ASM}^{\circ}(n, r)=\emptyset, \quad \text { for each } n, r \in \mathbb{P} \text { with } r<n .
$$

It can be seen immediately that the containment of (54) is in fact an equality for $n=1$ and $n=2$, and it follows from (63) in Section 8 that this is also the case for $n=3$.

We now give the main result for the enumeration of $\operatorname{ASM}(n, r)$ and $\operatorname{ASM}^{\circ}(n, r)$.

Theorem 2. For fixed $n \in \mathbb{P}$, there exists a function $A_{n}(r)$, the Ehrhart polynomial of the alternating sign matrix polytope $\mathcal{A}_{n}$, which satisfies:

(i) $A_{n}(r)$ is a polynomial in $r$ of degree $(n-1)^{2}$.

(ii) $|\operatorname{ASM}(n, r)|=A_{n}(r)$, for each $r \in \mathbb{N}$. 
(iii) $\left|\mathrm{ASM}^{\circ}(n, r)\right|=(-1)^{n+1} A_{n}(-r)$, for each $r \in \mathbb{N}$.

(iv) $A_{n}(-1)=A_{n}(-2)=\ldots=A_{n}(-n+1)=0$.

(v) $A_{n}(1)=\prod_{i=0}^{n-1} \frac{(3 i+1) !}{(n+i) !}$.

(vi) $A_{n}(r)$ can be expressed in the form $A_{n}(r)=\sum_{k=n-1}^{(n-1)^{2}} c_{k}\left(\begin{array}{c}r+k \\ (n-1)^{2}\end{array}\right)$, with $c_{k} \in \mathbb{N}$ for each $k \in\left[n-1,(n-1)^{2}\right]$.

Proof. All of the conclusions of this theorem follow straightforwardly from results already obtained or stated in this paper. First, the existence of an Ehrhart polynomial $\mathcal{L}_{\mathcal{A}_{n}}(r)=A_{n}(r)$ follows from the fact, implied by Theorem 1 , that $\mathcal{A}_{n}$ is an integral convex polytope. Properties (i)-(iii) in this theorem then follow from (i)-(iii) of (51) applied to $\mathcal{A}_{n}$, using (39), (40) and (52). Property (iv) follows from property (iii) applied to (55). Property (v) follows from property (ii) applied to (6). Finally, property (vi) follows from (iv) of (51), and from property (iv), which gives $c_{0}=c_{1}=\ldots=c_{n-2}=0$.

It follows that the explicit polynomial $A_{n}(r)$ for a particular $n \in \mathbb{P}$ can be found by interpolation using the $n+1$ values provided by $A_{n}(0)=1$ and properties (iv) and (v), together with $n^{2}-3 n+1$ further values obtained by the direct enumeration of cases of $\operatorname{ASM}(n, r)$ or $\operatorname{ASM}^{\circ}(n, r)$ and the application of properties (ii) and (iii). We have done this for $n=3,4$ and 5, some of the required values being provided in Table 1 . Together with the trivial cases $n=1$ and 2, the results, expressed in the form of (vi) of Theorem 2, are

$$
\begin{aligned}
A_{1}(r)= & \left(\begin{array}{c}
r \\
0
\end{array}\right), \quad A_{2}(r)=\left(\begin{array}{c}
r+1 \\
1
\end{array}\right), \quad A_{3}(r)=\left(\begin{array}{c}
r+2 \\
4
\end{array}\right)+2\left(\begin{array}{c}
r+3 \\
4
\end{array}\right)+\left(\begin{array}{c}
r+4 \\
4
\end{array}\right) \\
A_{4}(r)= & 3\left(\begin{array}{c}
r+3 \\
9
\end{array}\right)+80\left(\begin{array}{c}
r+4 \\
9
\end{array}\right)+415\left(\begin{array}{c}
r+5 \\
9
\end{array}\right)+592\left(\begin{array}{c}
r+6 \\
9
\end{array}\right)+253\left(\begin{array}{c}
r+7 \\
9
\end{array}\right)+ \\
& 32\left(\begin{array}{c}
r+8 \\
9
\end{array}\right)+\left(\begin{array}{c}
r+9 \\
9
\end{array}\right) \\
A_{5}(r)= & 70\left(\begin{array}{c}
r+4 \\
16
\end{array}\right)+14468\left(\begin{array}{c}
r+5 \\
16
\end{array}\right)+521651\left(\begin{array}{c}
r+6 \\
16
\end{array}\right)+6002192\left(\begin{array}{c}
r+7 \\
16
\end{array}\right)+ \\
& 28233565\left(\begin{array}{c}
r+8 \\
16
\end{array}\right)+61083124\left(\begin{array}{c}
r+9 \\
16
\end{array}\right)+64066830\left(\begin{array}{c}
r+10 \\
16
\end{array}\right)+ \\
& 32866092\left(\begin{array}{c}
r+11 \\
16
\end{array}\right)+7998192\left(\begin{array}{c}
r+12 \\
16
\end{array}\right)+854464\left(\begin{array}{c}
r+13 \\
16
\end{array}\right)+ \\
& 34627\left(\begin{array}{c}
r+14 \\
16
\end{array}\right)+412\left(\begin{array}{c}
r+15 \\
16
\end{array}\right)+\left(\begin{array}{c}
r+16 \\
16
\end{array}\right) .
\end{aligned}
$$


We note that the fact that (54) is an equality for $n=1,2$ and 3, implies that $\left|\operatorname{ASM}^{\circ}(n, r)\right|$ $=|\operatorname{ASM}(n, r-n)|$, for all $n \in\{1,2,3\}$ and $r \in \mathbb{P}$ with $r \geq n$, which in turn implies that $A_{n}(r)=(-1)^{n+1} A_{n}(-n-r)$, for all $n \in\{1,2,3\}$ and $r \in \mathbb{C}$, as can be observed in (56).

Finally, we now outline the results for $\mathcal{B}_{n}$ and $\operatorname{SMS}(n, r)$ which correspond to those of this section for $\mathcal{A}_{n}$ and $\operatorname{ASM}(n, r)$. Even though the results for $\mathcal{B}_{n}$ and $\operatorname{SMS}(n, r)$ are previously-known, having been conjectured in [2] and first proved in [32, 62], we state them here to show their similarity with those for $\mathcal{A}_{n}$ and $\operatorname{ASM}(n, r)$. The relative interior $\mathcal{B}_{n}^{\circ}$ of the Birkhoff polytope $\mathcal{B}_{n}$ is the set of positive real-entry $n \times n$ matrices for which all complete row and column sums are 1 , and $\operatorname{SMS}^{\circ}(n, r):=r \mathcal{B}_{n}^{\circ} \cap \mathbb{Z}^{n^{2}}$ is the set of positive integer-entry matrices for which all complete row and column sums are $r$. It follows that if $\operatorname{SMS}(n, r)$ and $\operatorname{SMS}^{\circ}(n, r)$ are substituted for $\operatorname{ASM}(n, r)$ and $\operatorname{ASM}^{\circ}(n, r)$ in (54) and (55), then the equations still hold, and furthermore that the containment in (54) can be replaced by an equality in all cases. Since $\mathcal{B}_{n}$ is an integral convex polytope, it has an Ehrhart polynomial $\mathcal{L}_{\mathcal{B}_{n}}(r)$, which we denote $H_{n}(r)$. Applying (51), and using (5), (39) and (40), it now follows that all of Theorem 2 still holds if the substitutions $H_{n}(r)$ for $A_{n}(r)$, 'Birkhoff polytope' for 'alternating sign matrix polytope', $\mathcal{B}_{n}$ for $\mathcal{A}_{n}, \operatorname{SMS}(n, r)$ for $\operatorname{ASM}(n, r)$, $\operatorname{SMS}^{\circ}(n, r)$ for $\operatorname{ASM}^{\circ}(n, r)$, and $n$ ! for $\prod_{i=0}^{n-1}(3 i+1) ! /(n+i)$ ! are made. Furthermore, the fact that the counterpart to (54) is now an equality implies that $\left|\operatorname{SMS}^{\circ}(n, r)\right|=\mid \operatorname{SMS}(n, r-$ $n) \mid$, for all $n, r \in \mathbb{P}$ with $r \geq n$, which leads to the further properties that $H_{n}(r)=$ $(-1)^{n+1} H_{n}(-n-r)$ for all $n \in \mathbb{P}$ and $r \in \mathbb{C}$, and, in the counterpart to (vi) of Theorem 2, that $c_{k}=c_{n(n-1)-k}$ for each $n \in \mathbb{P}$ and $k \in\left[n-1,(n-1)^{2}\right]$.

\section{Higher Spin Alternating Sign Matrices of Size 3}

In the previous section, the enumeration of higher spin alternating sign matrices was studied using a general, but nondirect, approach. In this section, we consider the special case of $3 \times 3$ higher spin alternating sign matrices, and provide a direct bijective derivation of the enumeration formula, from Theorem 2 and (56),

$$
|\operatorname{ASM}(3, r)|=\left(\begin{array}{c}
r+2 \\
4
\end{array}\right)+2\left(\begin{array}{c}
r+3 \\
4
\end{array}\right)+\left(\begin{array}{c}
r+4 \\
4
\end{array}\right), \text { for each } r \in \mathbb{N} .
$$

We begin by outlining a derivation of the corresponding semimagic squares formula,

$$
|\operatorname{SMS}(3, r)|=\left(\begin{array}{c}
r+2 \\
4
\end{array}\right)+\left(\begin{array}{c}
r+3 \\
4
\end{array}\right)+\left(\begin{array}{c}
r+4 \\
4
\end{array}\right) \text {, for each } r \in \mathbb{N},
$$

since the derivation of (57) will be similar. The polynomial (58) for $|\operatorname{SMS}(3, r)|$ was obtained directly in $[1,2,12,49,53]$. The derivation given here most closely follows that of $[12]$. 
Let $S_{3}$ be the set of permutations of 123 , and consider the set

$$
\begin{aligned}
& C(r):= \\
& \left\{\begin{array}{c|l}
a=\left(a_{123}, a_{132}, a_{213},\right. \\
\left.a_{231}, a_{312}, a_{321}\right) \in \mathbb{N}^{6} & \left.\begin{array}{l}
\bullet \sum_{\sigma \in S_{3}} a_{\sigma}=r \\
\bullet a_{132}=0 \text { or }\left(a_{321}=0 \text { and } a_{132} \neq 0\right) \\
\text { or }\left(a_{213}=0 \text { and } a_{132}, a_{321} \neq 0\right)
\end{array}\right\} .
\end{array}\right.
\end{aligned}
$$

Using the fact that, for any $m, n \in \mathbb{N}$, the number of tuples $\left(a_{1}, \ldots, a_{m+n}\right) \in \mathbb{P}^{m} \times \mathbb{N}^{n}$ with $\sum_{i=1}^{m+n} a_{i}=r$ is $\left(\begin{array}{c}r+n-1 \\ m+n-1\end{array}\right)$ (which can be obtained by considering tuples $\left(b_{1}, \ldots, b_{m+n-1}\right)$ $\in \mathbb{P}^{m+n-1}$ with $b_{1}<\ldots<b_{m} \leq b_{m+1} \leq \ldots \leq b_{m+n-1} \leq r$, and using the bijection $b_{i}=\sum_{j=1}^{i} a_{j}$ for each $\left.i \in[m+n-1]\right)$, it follows that

$$
|C(r)|=\left(\begin{array}{c}
r+4 \\
4
\end{array}\right)+\left(\begin{array}{c}
r+3 \\
4
\end{array}\right)+\left(\begin{array}{c}
r+2 \\
4
\end{array}\right)
$$

where the three binomial coefficients correspond respectively to the three alternatives in the second condition of (59).

Now let $P_{\sigma}$ be the permutation matrix given by $\left(P_{\sigma}\right)_{i j}:=\delta_{i, \sigma_{j}}$ for each $\sigma \in S_{3}$ and $i, j \in[3]$, and define, for each $a \in C(r)$,

$$
\theta(a):=\sum_{\sigma \in S_{3}} a_{\sigma} P_{\sigma}=\left(\begin{array}{lll}
a_{123}+a_{132} & a_{213}+a_{312} & a_{231}+a_{321} \\
a_{213}+a_{231} & a_{123}+a_{321} & a_{132}+a_{312} \\
a_{312}+a_{321} & a_{132}+a_{231} & a_{123}+a_{213}
\end{array}\right) .
$$

It can immediately be seen that $\theta(a) \in \operatorname{SMS}(3, r)$ for each $a \in C(r)$. It can also be checked straightforwardly that $\theta$ is a bijection between $C(r)$ and $\operatorname{SMS}(3, r)$, the inverse mapping being, for each $A \in \operatorname{SMS}(3, r)$,

$$
\theta^{-1}(A)= \begin{cases}\left(A_{11}, 0, A_{33}-A_{11}, A_{32}, A_{23}, A_{22}-A_{11}\right), & A_{11} \leq A_{22} \text { and } A_{11} \leq A_{33} \\ \left(A_{22}, A_{11}-A_{22}, A_{33}-A_{22}, A_{13}, A_{31}, 0\right), & A_{22}<A_{11} \text { and } A_{22} \leq A_{33} \\ \left(A_{33}, A_{11}-A_{33}, 0, A_{21}, A_{12}, A_{22}-A_{33}\right), & A_{33}<A_{11} \text { and } A_{33}<A_{22},\end{cases}
$$

where the three cases of (62) correspond respectively to the three alternatives in the second condition of (59). Therefore $|\operatorname{SMS}(3, r)|=|C(r)|$, so that (60) gives (58), thereby completing its bijective derivation.

Proceeding to $3 \times 3$ higher spin alternating sign matrices, we first note that $\operatorname{ASM}(3, r)$ is simply the set of $3 \times 3$ integer-entry matrices for which all complete row and column sums are $r$, and the eight external entries are nonnegative,

$$
\begin{aligned}
& \operatorname{ASM}(3, r)= \\
& \left\{A=\left(\begin{array}{lll}
A_{11} & A_{12} & A_{13} \\
A_{21} & A_{22} & A_{23} \\
A_{31} & A_{32} & A_{33}
\end{array}\right) \in \mathbb{Z}^{3 \times 3} \mid \begin{array}{l}
\bullet \sum_{j^{\prime}=1}^{3} A_{i j^{\prime}}=\sum_{i^{\prime}=1}^{3} A_{i^{\prime} j}=r \text { for all } i, j \in[3] \\
\bullet A_{i j} \geq 0 \text { for all }(i, j) \in[3] \times[3] /\{(2,2)\}
\end{array}\right\} .
\end{aligned}
$$


To obtain (63) from (1), it only needs to be verified that if $A$ is an element of the RHS of (63), then each partial sum $A_{12}+A_{22}, A_{21}+A_{22}, A_{23}+A_{22}$ and $A_{32}+A_{22}$ is nonnegative. For the case of $A_{12}+A_{22}, A_{12}+A_{22}+A_{32}=A_{31}+A_{32}+A_{33}(=r)$ gives $A_{12}+A_{22}=A_{31}+A_{33}$, but $A_{31} \geq 0$ and $A_{33} \geq 0$ now imply that $A_{12}+A_{22} \geq 0$ as required. The other three cases follow by symmetry.

The set of standard alternating sign matrices, $\operatorname{ASM}(3,1)$, is the set $\left\{P_{\sigma} \mid \sigma \in S_{3}\right\}$ of $3 \times 3$ permutation matrices, together with the matrix $\left(\begin{array}{rrr}0 & 1 & 0 \\ 1 & -1 & 1 \\ 0 & 1 & 0\end{array}\right)$ which will be denoted as $P_{0}$. Setting $S_{3}^{\prime}:=S_{3} \cup\{0\}$, we now define, in analogy with (59) and (61),

$$
\begin{aligned}
& C^{\prime}(r):= \\
& \left\{\begin{array}{c|l}
\bullet & \sum_{\sigma \in S_{3}^{\prime}} a_{\sigma}=r \\
a=\left(a_{123}, a_{132}, a_{213}, a_{231},\right. & a_{132}=a_{0}=0 \text { or } \\
\left.a_{312}, a_{321}, a_{0}\right) \in \mathbb{N}^{7} & \left(a_{321}=a_{0}=0 \text { and } a_{132} \neq 0\right) \text { or } \\
& \left(a_{213}=a_{0}=0 \text { and } a_{132}, a_{321} \neq 0\right) \text { or } \\
\left(a_{123}=a_{321}=0 \text { and } a_{0} \neq 0\right)
\end{array}\right\},
\end{aligned}
$$

and, for each $a \in C^{\prime}(r)$,

$$
\theta^{\prime}(a):=\sum_{\sigma \in S_{3}^{\prime}} a_{\sigma} P_{\sigma}=\left(\begin{array}{ccc}
a_{123}+a_{132} & a_{213}+a_{312}+a_{0} & a_{231}+a_{321} \\
a_{213}+a_{231}+a_{0} & a_{123}+a_{321}-a_{0} & a_{132}+a_{312}+a_{0} \\
a_{312}+a_{321} & a_{132}+a_{231}+a_{0} & a_{123}+a_{213}
\end{array}\right) .
$$

It immediately follows that

$$
\left|C^{\prime}(r)\right|=\left(\begin{array}{c}
r+4 \\
4
\end{array}\right)+2\left(\begin{array}{c}
r+3 \\
4
\end{array}\right)+\left(\begin{array}{c}
r+2 \\
4
\end{array}\right),
$$

and that $\theta^{\prime}(a) \in \operatorname{ASM}(3, r)$ for each $a \in C^{\prime}(r)$. It can also be checked, using (63) and the bijection (61) and (62) between $C(r)$ and $\operatorname{SMS}(3, r)$, that $\theta^{\prime}$ is a bijection between $C^{\prime}(r)$ and $\operatorname{ASM}(3, r)$, the inverse mapping being, for each $A \in \operatorname{ASM}(3, r)$,

$$
\begin{aligned}
& \theta^{\prime-1}(A)= \\
& \begin{cases}\left(A_{11}, 0, A_{33}-A_{11}, A_{32}, A_{23}, A_{22}-A_{11}, 0\right), & A_{11} \leq A_{22} \text { and } A_{11} \leq A_{33} \\
\left(A_{22}, A_{11}-A_{22}, A_{33}-A_{22}, A_{13}, A_{31}, 0,0\right), \quad 0 \leq A_{22}<A_{11} \text { and } A_{22} \leq A_{33} \\
\left(A_{33}, A_{11}-A_{33}, 0, A_{21}, A_{12}, A_{22}-A_{33}, 0\right), \quad A_{33}<A_{11} \text { and } A_{33}<A_{22} \\
\left(0, A_{11}, A_{33}, A_{13}, A_{31}, 0,-A_{22}\right), \quad A_{22}<0,\end{cases}
\end{aligned}
$$

where the four cases of (67) correspond respectively to the four alternatives in the second condition of (64). Therefore $|\operatorname{ASM}(3, r)|=\left|C^{\prime}(r)\right|$, so that (66) gives (57), thereby completing its bijective derivation. 
In this section, particular sets $C(r)$ and $C^{\prime}(r)$ of weak compositions of $r$ into six and seven parts, with at most five parts nonzero, have been found which are in bijection with $\operatorname{SMS}(3, r)$ and $\operatorname{ASM}(3, r)$ respectively, where the mapping from a weak composition to a matrix simply uses the parts of the composition as coefficients in a linear combination of permutation matrices or standard alternating sign matrices. It would clearly be worth investigating whether these results can be generalized to larger integers $n$ to give sets of weak compositions of $r$ into $n$ ! and $\prod_{i=0}^{n-1}(3 i+1) ! /(n+i)$ ! parts, with at most $(n-1)^{2}+1$ parts nonzero, which are similarly in bijection with $\operatorname{SMS}(n, r)$ and $\operatorname{ASM}(n, r)$ respectively. This, however, seems to be a challenging problem.

\section{Discussion}

We conclude this paper by outlining some possible directions for further research. While all of these can be simply stated, and follow from the content of this paper in obvious ways, some may well turn out to be of limited interest, or to lead to prohibitively-difficult problems.

In this paper, we studied the configurations of certain integrable statistical mechanical models with domain wall boundary conditions. One area for additional work would therefore be the consideration of further integrable statistical mechanical models with

boundary conditions of domain-wall-type. A possible component of such work would be the derivation of determinant formulae for the partition functions of such models, this having already been done for certain models in [19, 30].

In Section 3, we discussed the determinant formula for higher spin integrable vertex models. Although it seems unlikely that this formula can be used for the unweighted enumeration of higher spin alternating sign matrices or semimagic squares, it may still be possible for it to be used to obtain certain weighted enumeration formulae. More specifically, each vertex type of $\mathcal{V}(r)$, as defined in (12), would be given a weight, and the overall weight of a higher spin alternating sign matrix would be the product of the weights of all the vertex types associated with the corresponding edge matrix pair. Certain such weighted enumeration formulae are already known for standard alternating sign matrices (see for example $[22,33,47]$ ). In these cases, vertex type (4) in Figure 3 (which corresponds to each entry of -1 in a standard alternating sign matrix) is weighted by 2 or 3 , and the other five vertex types of $\mathcal{V}(1)$ are each weighted by 1 .

In Sections 4 and 5, we considered various other combinatorial objects, namely certain sets of lattice paths, monotone triangles, corner sum matrices and complementary edge matrix pairs, which are in simple bijection with higher spin alternating sign matrices. 
Each of these representations could be studied further, and might provide useful statistics according to which higher spin alternating sign matrices could be weighted and classified. The fully packed loop configurations defined in Section 5 also seem worthy of further study, especially since their classification according to the link pattern formed among the external points by the open paths, may provide an interesting generalization of such a classification for the standard case of $r=1$ (see for example [20, 21, 28, 29, 68, 75]).

It is also common to impose further conditions on semimagic squares (see for example [6] and [63, Proposition 4.6.21]) and standard alternating sign matrices (see for example [47, $54,56,57,58])$. It would therefore seem natural to investigate the effects of imposing similar such conditions on higher spin alternating sign matrices, it being expected that this would lead to the introduction of further, not necessarily integral, convex polytopes.

Semimagic squares are a special case of contingency or frequency tables (see for example [38]), these simply being nonnegative integer-entry rectangular matrices with arbitrary prescribed row and column sums. Similar generalizations of higher spin alternating sign matrices in which the complete row and column sums are prescribed, but not necessarily all equal, and the matrices are not necessarily square, would thus provide another direction for further study.

In Section 6, we defined the alternating sign matrix polytope, and showed that its vertices are standard alternating sign matrices. Many additional results for this polytope, including the enumeration of its facets, the characterization of its face lattice, and the description of its projection to the permutohedron have been obtained in [65], but this polytope could still be investigated further.

In Section 7, we obtained certain enumeration formulae for higher spin alternating sign matrices with fixed size $n$ and variable line-sum $r$, but we have not obtained formulae for the case of variable $n$ and fixed $r$. Formulae for semimagic squares with variable $n$, and $r=2$ or $r=3$ are known (see for example [2], [13, Ch. 9], [23, Ex. 25, Page 124] and [64, Sec. 5.5], but note that the $r=3$ formula in [23] contains misprints). However, it might be expected that the difference in difficulties in obtaining, say, the $r=2$ semimagic square formula and an $r=2$ higher spin alternating sign matrix formula, might be comparable to the substantial difference in difficulties in obtaining the $r=1$ formulae (5) and (6).

Finally, as indicated at the end of Section 8, it would be interesting to see whether the approach used there can be generalized to give bijective derivations of enumeration formulae for semimagic squares or higher spin alternating sign matrices with fixed size larger than 3 . 


\section{Acknowledgements}

We thank Omar Foda and Jessica Striker for correspondence regarding their papers [18] and [65]. VK is supported by an EPSRC-funded postgraduate scholarship.

\section{References}

[1] M. Abramson and W. O. J. Moser Discrete Math. 6 (1973) 1-14 Arrays with Fixed Row and Column Sums

[2] H. Anand, V. C. Dumir and H. Gupta Duke Math. J. 33 (1966) 757-769 A Combinatorial Distribution Problem

[3] G. Andrews Invent. Math. 53 (1979) 193-225 Plane Partitions III: The Weak Macdonald Conjecture

[4] G. Andrews J. Combin. Theory Ser. A 66 (1994) 28-39 Plane Partitions V: The TSSCPP Conjecture

[5] R. J. Baxter Exactly Solved Models in Statistical Mechanics (Academic Press, 1982)

[6] M. Beck, M. Cohen, J. Cuomo and P. Gribelyuk Amer. Math. Monthly 110 (2003) 707-717 The Number of "Magic" Squares, Cubes, and Hypercubes

[7] M. Beck and S. Robins Computing the Continuous Discretely: Integer-Point Enumeration in Polyhedra (Springer, 2007)

[8] R. E. Behrend Determinant Formula for the Partition Function of Higher Spin Vertex Models with Domain Wall Boundary Conditions (unpublished notes)

[9] R. E. Behrend arXiv:math/0701755 Osculating Paths and Oscillating Tableaux

[10] G. Birkhoff Univ. Nac. Tucumán. Revista A 5 (1946) 147-151 Tres Observaciones sobre el Algebra Lineal

[11] N. M. Bogoliubov, A. G. Pronko and M. B. Zvonarev J. Phys. A 35 (2002) 5525-5541 Boundary Correlation Functions of the Six-Vertex Model

[12] M. Bóna Math. Mag. 70 (1997) 201-203 A New Proof of the Formula for the Number of $3 \times 3$ Magic Squares

[13] M. Bóna Introduction to Enumerative Combinatorics (Mc Graw-Hill, 2007)

[14] M. Bousquet-Mélou and L. Habsieger Discrete Math. 139 (1995) 57-72 Sur les Matrices à Signes Alternants

[15] R. Brak Osculating Lattice Paths and Alternating Sign Matrices in Proceedings of 9th Formal Power Series and Algebraic Combinatorics Conference (Vienna, 1997) Available at http://www.ms.unimelb.edu.au/ ${ }^{\sim}$ brak/publications.html 
[16] D. M. Bressoud Proofs and Confirmations: The Story of the Alternating Sign Matrix Conjecture (Cambridge University Press, 1999)

[17] D. M. Bressoud and J. Propp Notices Amer. Math. Soc. 46 (1999) 637-646 How the Alternating Sign Matrix Conjecture was Solved

[18] A. Caradoc, O. Foda and N. Kitanine J. Stat. Mech. (2006) P03012 18pp. Higher Spin Vertex Models with Domain Wall Boundary Conditions

[19] A. Caradoc, O. Foda, M. Wheeler and M. Zuparic J. Stat. Mech. (2007) P03010 14pp. On the Trigonometric Felderhof Model with Domain Wall Boundary Conditions

[20] F. Caselli and C. Krattenthaler J. Combin. Theory Ser. A 108 (2004) 123-146 Proof of Two Conjectures of Zuber on Fully Packed Loop Configurations

[21] F. Caselli, C. Krattenthaler, B. Lass and P. Nadeau Electron. J. Combin. 11(2) (2005) R16 43pp. On the Number of Fully Packed Loop Configurations with a Fixed Associated Matching

[22] F. Colomo and A. G. Pronko J. Stat. Mech. (2005) P01005 33pp. Square Ice, Alternating Sign Matrices and Classical Orthogonal Polynomials

[23] L. Comtet Advanced Combinatorics: The Art of Finite and Infinite Expansions (Reidel, 1974)

[24] J. de Gier Discrete Math. 298 (2005) 365-388 Loops, Matchings and Alternating Sign Matrices

[25] J. de Gier J. Stat. Mech. (2007) N02001 6pp. The Razumov-Stroganov Conjecture: Stochastic Processes, Loops and Combinatorics

[26] P. Di Francesco J. Stat. Mech. (2006) P09008 14pp. Totally Symmetric SelfComplementary Plane Partitions and the Quantum Knizhnik-Zamolodchikov Equation: A Conjecture

[27] P. Di Francesco J. Stat. Mech. (2007) P01024 22pp. Open Boundary Quantum Knizhnik-Zamolodchikov Equation and the Weighted Enumeration of Plane Partitions with Symmetries

[28] P. Di Francesco, P. Zinn-Justin and J.-B. Zuber Electron. J. Combin. 11(1) (2004) R64 11pp. A Bijection Between Classes of Fully Packed Loops and Plane Partitions

[29] P. Di Francesco and J.-B. Zuber J. Stat. Mech. (2004) P06005 20pp. On Fully Packed Loop Configurations with Four Sets of Nested Arches

[30] A. Dow and O. Foda J. Stat. Mech. (2006) P05010 16pp. On the Domain Wall Partition Functions of Level-1 Affine so(n) Vertex Models

[31] O. Eğecioğlu, T. Redmond and C. Ryavec Electron. J. Combin. 8 (2001) R36 51 pp. From a Polynomial Riemann Hypothesis to Alternating Sign Matrices 
[32] E. Ehrhart C. R. Acad. Sci. Paris Sér. A 277 (1973) 651-654 Sur les Carrés Magiques

[33] N. Elkies, G. Kuperberg, M. Larsen and J. Propp J. Algebraic Combin. 1 (1992) 111-132 \& 219-234 Alternating-Sign Matrices and Domino Tilings (Parts I \& II)

[34] I. Fischer Adv. Appl. Math. 37 (2006) 249-267 The Number of Monotone Triangles with Prescribed Bottom Row

[35] I. Fischer J. Combin. Theory Ser. A 114 (2007) 253-264 A New Proof of the Refined Alternating Sign Matrix Theorem

[36] J. Fuchs Affine Lie Algebras and Quantum Groups (Cambridge University Press, 1992)

[37] J. Fuchs and C. Schweigert Symmetries, Lie Algebras and Representations (Cambridge University Press, 1997)

[38] M. Gail and N. Mantel J. Amer. Stat. Assoc. 72 (1977) 859-862 Counting the Number of $r \times c$ Contingency Tables with Fixed Margins

[39] C. Gómez, M. Ruiz-Altaba and G. Sierra Quantum Groups in Two-Dimensional Physics (Cambridge University Press, 1996)

[40] M. Idzumi, T. Tokihiro and M. Arai J. Physique I 4 (1994) 1151-1159 Solvable Nineteen-Vertex Models and Quantum Spin Chains of Spin One

[41] M. Ishikawa arXiv:math/0602068 On Refined Enumerations of Totally Symmetric Self-Complementary Plane Partitions I

[42] M. Ishikawa arXiv:math/0606082 On Refined Enumerations of Totally Symmetric Self-Complementary Plane Partitions II

[43] A. G. Izergin Soviet Phys. Dokl. 32 (1987) 878-879 Partition Function of a SixVertex Model in a Finite Volume

[44] A. G. Izergin, D. A. Coker and V. E. Korepin J. Phys. A 25 (1992) 4315-4334 Determinant Formula for the Six-Vertex Model

[45] V. E. Korepin Comm. Math. Phys. 86 (1982) 391-418 Calculation of Norms of Bethe Wave Functions

[46] P. P. Kulish, N. Y. Reshetikhin and E. K. Sklyanin Lett. Math. Phys. 5 (1981) 393-403 Yang-Baxter Equation and Representation Theory: I

[47] G. Kuperberg Int. Math. Res. Not. (1996) 139-150 Another Proof of the AlternatingSign Matrix Conjecture

[48] G. Kuperberg Ann. of Math. 156 (2002) 835-866 Symmetry Classes of AlternatingSign Matrices under One Roof

[49] P. A. MacMahon Combinatory Analysis (Cambridge University Press, 1915 \& 1916) 
[50] W. H. Mills, D. P. Robbins and H. Rumsey Invent. Math. 66 (1982) 73-87 Proof of the Macdonald Conjecture

[51] W. H. Mills, D. P. Robbins and H. Rumsey J. Combin. Theory Ser. A 34 (1983) 340-359 Alternating Sign Matrices and Descending Plane Partitions

[52] W. H. Mills, D. P. Robbins and H. Rumsey J. Combin. Theory Ser. A 42 (1986) 277-292 Self-Complementary Totally Symmetric Plane Partitions

[53] G. B. Nath and P. V. K. Iyer J. Aust. Math. Soc. 14 (1972) 264-268 Note on the Combinatorial Formula for ${ }_{n} H_{r}$

[54] S. Okada J. Algebraic Combin. 23 (2006) 43-69 Enumeration of Symmetry Classes of Alternating Sign Matrices and Characters of Classical Groups

[55] J. Propp Discrete Math. Theor. Comput. Sci. Proceedings AA (DM-CCG) (2001) 43-58 The Many Faces of Alternating-Sign Matrices

[56] A. V. Razumov and Y. G. Stroganov Theor. Math. Phys. 141 (2004) 1609-1630 On Refined Enumerations of Some Symmetry Classes of ASMs

[57] D. P. Robbins Math. Intelligencer 13 (1991) 12-19 The Story of 1, 2, 7, 42, 429, $7436, \ldots$

[58] D. P. Robbins arXiv:math/0008045 Symmetry Classes of Alternating Sign Matrices

[59] D. P. Robbins and H. Rumsey Adv. Math. 62 (1986) 169-184 Determinants and Alternating Sign Matrices

[60] K. Sogo, Y. Akutsu and T. Abe Prog. Theor. Phys. 70 (1983) 730-738 New Factorized S-Matrix and its Application to Exactly Solvable q-State Model. I

[61] J. H. Spencer Amer. Math. Monthly 87 (1980) 397-399 Counting Magic Squares

[62] R. P. Stanley Duke Math. J 40 (1973) 607-632 Linear Homogeneous Diophantine Equations and Magic Labelings of Graphs

[63] R. P. Stanley Enumerative Combinatorics: Volume 1 (Cambridge University Press, 1986)

[64] R. P. Stanley Enumerative Combinatorics: Volume 2 (Cambridge University Press, 1999)

[65] J. Striker arXiv:0705.0998 The Alternating Sign Matrix Polytope

[66] U. Tamm Electron. J. Combin. 8 (2001) A1 31pp. Some Aspects of Hankel Matrices in Coding Theory and Combinatorics

[67] J. von Neumann A Certain Zero-Sum Two-Person Game Equivalent to the Optimal Assignment Problem in Contributions to the Theory of Games Vol. 2. Annals of Mathematics Studies No. 28 (Princeton University Press, 1953) 5-12

[68] B. Wieland Electron. J. Combin. 7 (2000) R37 13pp. Large Dihedral Symmetry of the Set of Alternating Sign Matrices 
[69] A. B. Zamolodchikov and V. A. Fateev Soviet J. Nuclear Phys. 32 (1980) 581-590 A Model Factorized S-matrix and an Integrable Spin-1 Heisenberg Chain

[70] D. Zeilberger Electron. J. Combin. 3 (1996) R13 84pp. Proof of the Alternating Sign Matrix Conjecture

[71] D. Zeilberger New York J. Math. 2 (1996) 59-68 Proof of the Refined Alternating Sign Matrix Conjecture

[72] D. Zeilberger Adv. Appl. Math. 34 (2005) 939-954 Dave Robbins' Art of Guessing

[73] G. M. Ziegler Lectures on Polytopes (Springer, 1995)

[74] P. Zinn-Justin Comm. Math. Phys. 272 (2007) 661-682 Combinatorial Point for Fused Loop Models

[75] J.-B. Zuber Electron. J. Combin. 11(1) (2004) R13 15pp. On the Counting of Fully Packed Loop Configurations: Some New Conjectures 